\title{
VOLUME PRESERVING MEAN CURVATURE FLOWS NEAR STRICTLY STABLE SETS IN FLAT TORUS
}

\author{
JOONAS NIINIKOSKI
}

\begin{abstract}
In this paper we establish a new stability result for smooth volume preserving mean curvature flows in flat torus $\mathbb{T}^{n}$ in dimensions $n=3,4$. The result says roughly that if an initial set is near to a strictly stable set in $\mathbb{T}^{n}$ in $H^{3}$-sense, then the corresponding flow has infinite lifetime and converges exponentially fast to a translate of the strictly stable (critical) set in $W^{2,5}$-sense.
\end{abstract}

\section{Contents}

1. Introduction 1

2. Preliminaries 2

3. $L^{2}$-monotonicity 10

4. The main result 17

5. Appendix 20

Acknowledgments 25

References 25

\section{INTRODUCTION}

A smooth evolution of sets $\left(E_{t}\right)_{t}$, that is, a smooth flow in $\mathbb{R}^{n}$ is a volume preserving mean curvature flow (VMCF), if for every time $t$ the (outer) normal velocity of the flow on $\partial E_{t}$ obeys the law

$$
V_{t}=\bar{H}_{t}-H_{t}
$$

where $H_{t}$ is the (scalar) mean curvature on $\partial E_{t}$ and $\bar{H}_{t}$ its integral average over $\partial E_{t}$. As the name suggests, a VMCF preserves the volume, which is in the contrast to a classical mean curvature flow with a smooth and bounded initial set. Such a flow shrinks the initial volume to zero in finite time.

The short time existence for a smooth VMCF in $\mathbb{R}^{n}$ is well-known. For any smooth (a closed set with a smooth boundary) and compact set $E \subset \mathbb{R}^{n}$ with $n \geq 2$ there is a unique VMCF starting from $E$. However, a VMCF $\left(E_{t}\right)_{t}$ may develop singularities such as self-intersections of the boundary $\partial E_{t}$ within a finite time, see [17]. Another type of singularities of VMCF in a free boundary setting is studied in [5], where it is shown that certain thin necks have to pinch-off under VMCF. A natural problem is to find a sufficient condition for the initial set such that the VMCF starting from the set does not form singularities and has infinite lifetime.

Several contributions concerning the previous question have been made over the years. The classical result of Huisken [15] (see also Gage [11] in the case $n=2$ ) says that for any smooth, compact and convex set $E \subset \mathbb{R}^{n}$ there is a unique $\mathrm{VMCF}\left(E_{t}\right)_{t}$ starting from $E$ such that the flow has infinite lifetime and converges exponentially fast to a closed ball of the volume $|E|$ in $C^{\infty}$-topology. In the dimension $n=3$, the result by Li [16] gives us an alternative condition for a connected initial boundary based on a certain energy such that the corresponding VMCF has infinite lifetime and converges exponentially fast to a ball. Notice that if VMCF converges in $C^{2}$-sense, then the limit set is a finite union of balls with mutually positive distance. This follows from the Alexandrov theorem.

This naturally raises questions about the stability of VMCF near stable sets, in this case the closed Euclidean balls. Such problems are often called stability problems. Escher and Simonett [9] used to center manifold analysis to prove that if $E \subset \mathbb{R}^{n}$ is a smooth compact set and $\bar{B}(x, r)$ is a

2010 Mathematics Subject Classification. Primary 53C44. Secondary 35K93.

Key words and phrases. Periodic stability, strictly stable sets, volume preserving mean curvature flow. 
closed ball with the same volume such that $\partial E$ is $C^{1, \alpha}$-close to $\partial B(x, r)$, then the VMCF starting from $E$ has infinite lifetime and converges to a translate of $\bar{B}(x, r)$ exponentially fast in $C^{k}$-sense for any $k \in \mathbb{N}$.

Instead of having generic smooth sets in $\mathbb{R}^{n}$, we may focus on periodic smooth sets, that is, the smooth sets in $\mathbb{R}^{n}$ invariant under the lattice translations. This again leads us to consider the flat torus $\mathbb{T}^{n}$ in place of $\mathbb{R}^{n}$. One motivation for this is that there then are more different types of compact and critical sets than in $\mathbb{R}^{n}$. Also, the notion of VMCF generalizes to the flat torus and corresponds to the periodic VMCFs in $\mathbb{R}^{n}$. We are interested in the subclass of compact and critical sets in $\mathbb{T}^{n}$ called strictly stable sets (with respect to perimeter), see Definition 2.12. Examples of strictly stable sets (besides single balls, cylinders and strips) in $\mathbb{T}^{3}$ are those sets having a Schwarz surface as a boundary, see [18]. Acerbi, Fusco and Morini prove that strictly stable sets in $\mathbb{T}^{n}$ are always isolated local perimeter minimizers (under the notion of volume in $\mathbb{T}^{n}$ ), see [2, Theorem 1.1]. In contrast, the only smooth local perimeter minimizers in $\mathbb{R}^{n}$ are just the single balls. This essentially follows from [7], see also [19].

Our goal is to prove the following stability result for VMCFs near strictly stable sets in the flat torus $\mathbb{T}^{n}$ with $n=3,4$ using the notion of graph surface representation in normal direction and Sobolev spaces on smooth compact hypersurfaces.

\section{Theorem (Main result).}

Let $F \subset \mathbb{T}^{n}$, where $n=3,4$, be a strictly stable set and let $\nu_{F}$ be the unit normal of $\partial F$ with the inside-out orientation. There exists a positive constant $\delta_{0} \in \mathbb{R}_{+}$depending on $F$ such that the following hold.

If $E_{0}$ is a smooth set in $\mathbb{T}^{n}$ with the same volume as $F$ and having a boundary of the form

$$
\partial E_{0}=\left\{x+\psi_{0}(x) \nu_{F}(x): x \in \partial F\right\},
$$

where $\psi_{0} \in C^{\infty}(\partial F)$ and $\left\|\psi_{0}\right\|_{H^{3}(\partial F)} \leq \delta_{0}$, then the VMCF $\left(E_{t}\right)_{t}$ starting from $E_{0}$ has infinite lifetime and converges to a translate $F+p$ of $F$ exponentially fast in $W^{2,5}$-sense from the point of view of the boundary $\partial(F+p)$. Moreover, $|p| \rightarrow 0$ as $\left\|\psi_{0}\right\|_{H^{3}(\partial F)} \rightarrow 0$.

\section{Remarks.}

Of course, using the same arguments we obtain a similar result in $\mathbb{T}^{2}$. Since the convergence happens exponentially fast in time, the flow is also said to be exponentially stable near strictly stable set.

In terms of methods we are motivated by the paper Acerbi, Fusco, Julin and Morini [1], where they prove similar kinds of stability results for other volume preserving flows, namely the modified Mullins-Sekerka flow and the surface diffusion flow in the three dimensional flat torus $\mathbb{T}^{3}$. The cornerstone of our analysis (see Section 3) is to prove that $H^{3}$-closeness to a strictly stable set implies for the $\operatorname{VMCF}\left(E_{t}\right)_{t}$ that the $L^{2}$-norm of the normal velocity over $\partial E_{t}$, that is $\| \bar{H}_{t}-$ $H_{t} \|_{L^{2}\left(\partial E_{t}\right)}$, is decaying exponentially in time while the $L^{2}$-norm of its tangential gradient over $\partial E_{t}$ is bounded in time. In proving this we are heavily dependent on Sobolev interpolation inequalities, which is the reason we have to restrict ourselves to low dimensions.

\section{Preliminaries}

Flat torus. Recall that for given $n \geq 2$ the (unit) flat torus $\mathbb{T}^{n}$ is defined as the quotient space $\mathbb{R}^{n} / \mathbb{Z}^{n}$. Here the equivalence relation is given in the obvious way: $x \sim y$ exactly when $x-y \in \mathbb{Z}^{n}$. Functions $f: \mathbb{T}^{n} \rightarrow \mathbb{R}^{d}$ (for $d \in \mathbb{N}$ ) can be (canonically) identified with the class of $\mathcal{D}_{n}$-periodic maps $\mathbb{R}^{n} \rightarrow \mathbb{R}^{d}$, where $\mathcal{D}_{n}=\left[0,1\left[^{n}\right.\right.$ is the dyadic unit cube. The continuous maps $\phi: \mathbb{T}^{n} \rightarrow \mathbb{T}^{n}$ are identified (up to integer translations) with their continuous lifts $\tilde{\phi}: \mathbb{R}^{n} \rightarrow \mathbb{R}^{n}$. Such lifts $\tilde{\phi}: \mathbb{R}^{n} \rightarrow \mathbb{R}^{n}$ are of the form $\tilde{\phi}=L+u$, where $L \in M_{n}(\mathbb{Z})$ and $u$ is continuous and $\mathcal{D}_{n}$-periodic. Further, the sets in $\mathbb{T}^{n}$ can be identified with $\mathcal{D}_{n}$-periodic sets in $\mathbb{R}^{n}$. If $x \in \mathbb{T}^{n}$, then for any $p \in \mathbb{R}^{n}$ the notation $x+p$ means the element $q(\tilde{x}+p) \in \mathbb{T}^{n}$, where $\tilde{x} \in q^{-1}(x)$ and $q: \mathbb{R}^{n} \rightarrow \mathbb{T}^{n}$ is the quotient map.

We consider $\mathbb{T}^{n}$ as a smooth and compact manifold where the smooth structure is given via the quotient map $\mathbb{R}^{n} \rightarrow \mathbb{R}^{n} / \mathbb{Z}^{n}$. The natural flat Riemannian metric on $\mathbb{T}^{n}$ is induced by the standard Euclidean inner product $\langle\cdot, \cdot\rangle$ of $\mathbb{R}^{n}$ via the quotient map. Indeed, one can think $\mathbb{T}^{n}$ is "locally" the Euclidean $\mathbb{R}^{n}$. A compatible distance $d_{\mathbb{T}^{n}}: \mathbb{T}^{n} \times \mathbb{T}^{n} \rightarrow[0, \infty[$ is given by

$$
d_{\mathbb{T}^{n}}(x, y)=\min \left\{|\tilde{x}-\tilde{y}|: \tilde{x} \in q^{-1}(x), \tilde{y} \in q^{-1}(y)\right\} .
$$


For every Borel set in $A$ in $\mathbb{T}^{n}$ its $s$-dimensional Hausdorff measure $\mathcal{H}^{s}(A)$ is defined to be the corresponding Hausdorff-measure of the intersection of the periodic extension and $\mathcal{D}_{n}$.

A function $f: \mathbb{T}^{n} \rightarrow \mathbb{R}^{d}$ is locally $C^{k, \alpha}$-regular at $x \in \mathbb{T}^{n}$ exactly when its periodic extension $\tilde{f}$ to $\mathbb{R}^{n}$ is locally $C^{k, \alpha}$-regular at every representative $\tilde{x}$ of $x$. Thus, we set the $j$-th derivative $\mathrm{D}^{j} f(x)$ at $x$ to be $\mathrm{D}^{j} \tilde{f}(\tilde{x})$ for every $1 \leq j \leq k$. Again, a continuous map $\phi: \mathbb{T}^{n} \rightarrow \mathbb{T}^{n}$ is $C^{k, \alpha}$-regular exactly when its continuous lifts $\tilde{\phi}$ are $C^{k, \alpha}$-maps. Then $\phi$ has a unique continuous lift modulo integer translations so we may again see the derivatives of $\phi$ as the derivatives of its continuous lifts. If a diffeomorphism $\Phi: \mathbb{T}^{n} \rightarrow \mathbb{T}^{n}$ is close enough to the identity map id $\mathbb{T}^{n}$ in the sense that $\sup _{\mathbb{T}^{n}} d_{\mathbb{T}^{n}}\left(\Phi, \mathrm{id}_{\mathbb{T}^{n}}\right)$ is sufficiently small, then there is a unique diffeomorphic lift $\tilde{\Phi}$ such that $\tilde{\Phi}=\mathrm{id}+u$ and $\sup _{\mathbb{T}^{n}} d_{\mathbb{T}^{n}}\left(\Phi, \operatorname{id}_{\mathbb{T}^{n}}\right)=\sup _{\mathbb{R}^{n}}|u|$. In such a case, we set for every $l \in \mathbb{N}$ and $0 \leq \alpha \leq 1$ (for which $\Phi$ is $C^{l, \alpha}$-regular)

$$
\left\|\Phi-\operatorname{id}_{\mathbb{T}^{n}}\right\|_{C^{l, \alpha}\left(\mathbb{T}^{n} ; \mathbb{T}^{n}\right)}=\|u\|_{C^{l, \alpha}\left(\mathbb{R}^{n} ; \mathbb{R}^{n}\right)} .
$$

Further, we denote $\mathrm{id}_{\mathbb{T}^{n}}$ by id when there is no danger of confusion.

Smooth hypersurfaces. By a hypersurface, we mean an embedded submanifold of codimension 1 without boundary in our discussion. A set $\Gamma \subset \mathbb{T}^{n}$ is a $C^{k, \alpha}$-hypersurface exactly when its $\mathcal{D}_{n^{-}}$ periodic extension is a $C^{k, \alpha}$-hypersurface in $\mathbb{R}^{n}$. From now on, $\Sigma$ denotes a smooth and compact hypersurface of $\mathbb{T}^{n}$. Note that then $\Sigma$ has finitely many (path) connected components $\Sigma_{1}, \ldots, \Sigma_{N}$ which are in their own right smooth and compact hypersurfaces. The shorthand notation $|\Sigma|=$ $\mathcal{H}^{n-1}(\Sigma)$ is used from time to time without any further mention.

A function $f$ belongs to $C^{k}\left(\Sigma ; \mathbb{R}^{d}\right)$ if and only if it admits a $C^{k}$-extension to some open neighborhood of $\Sigma$. For every $x \in \Sigma$ the geometric tangent space $G_{x} \Sigma$ is defined as a unique $n-1$ dimensional subspace of $\mathbb{R}^{n}$ such that $x+G_{x} \Sigma$ is the tangent plane of $\Sigma$ at $x$. Equivalently we can set $G_{x} \Sigma=\mathrm{D} \phi\left(\mathbb{R}^{n-1}\right)$, where $\phi: U \rightarrow \mathbb{T}^{n}$ is any local parametrization of $\Sigma$ at $x$. Moreover, the orthogonal projection from $\mathbb{R}^{n}$ onto $G_{x} \Sigma$ is denoted by $P_{\Sigma}(x)$. Then $P_{\Sigma}: \Sigma \rightarrow \mathcal{L}\left(\mathbb{R}^{n} ; \mathbb{R}^{n}\right)$ is a smooth map.

For any $x \in \Sigma$, open neighborhood $U$ of $x$ in $\mathbb{T}^{n}$ and $f \in C^{1}\left(U ; \mathbb{R}^{k}\right)$ the tangential differential $\mathrm{D}_{\tau} f(x): \mathbb{R}^{n} \rightarrow \mathbb{R}^{k}$ of $f$ with respect to $\Sigma$ at $x$ is given by

$$
\mathrm{D}_{\tau} f(x)=\mathrm{D} f(x) P_{\Sigma}(x) .
$$

The definition does not depend on how $f$ is extended beyond $\Sigma$. In the case $k=1$ the dual of $\mathrm{D}_{\tau} f(x)$ is called the tangential gradient of $f$ with respect to $\Sigma$ at $x$ denoted by $\nabla_{\tau} f(x)$. Then we can write $\nabla_{\tau} f(x)=P_{\Sigma}(x) \nabla f(x)$ so $\nabla_{\tau} f(x) \in G_{x} \Sigma$. Further, in the case $k=n$ the tangential divergence $\operatorname{div}_{\tau} f(x)$ of $f$ with respect to $\Sigma$ at $x$ is defined as the trace of $\mathrm{D}_{\tau} f(x)$. These operations behave as their ordinary counterparts in $\mathbb{T}^{n}$.

Since $\Sigma$ has finitely many connected components, then it is also orientable, i.e., it admits a smooth unit normal field $\nu: \Sigma \rightarrow \mathbb{R}^{n}$, where $\nu(x) \in N_{x} \Sigma:=G_{x} \Sigma^{\perp}$ for every $x \in \Sigma$. The pair $(\Sigma, \nu)$ is called an oriented hypersurface $\Sigma$ with an orientation $\nu$. If $\Sigma^{\prime}$ is a $C^{1}$-hypersurface and $\Phi: \Sigma \rightarrow \Sigma^{\prime}$ is a $C^{1}$ diffeomorphism, then we have the change of variable formula. For any $h \in L^{1}\left(\Sigma^{\prime}, \mathcal{H}^{n-1}\right)$

$$
\int_{\Sigma^{\prime}} h \mathrm{~d} \mathcal{H}^{n-1}=\int_{\Sigma}(h \circ \Phi) J_{\tau} \Phi \mathrm{d} \mathcal{H}^{n-1}
$$

where the tangential Jacobian $J_{\tau} \Phi$ is given by $J_{\tau} \Phi=|\operatorname{det} \mathrm{D} \tilde{\Phi}|\left|(\mathrm{D} \tilde{\Phi})^{-\mathrm{T}} \nu\right|$ and $\tilde{\Phi}$ is any diffeomorphic extension of $\Phi$. This is independent of the choice of orientation.

The second fundamental form $B(x)$ of $\Sigma$ associated with the orientation $\nu$ at $x$ can be seen as the linear operator from $G_{x} \Sigma$ to itself or equivalently $\mathbb{R}^{n} \rightarrow \mathbb{R}^{n}$ given by

$$
B(x)=\mathrm{D}_{\tau} \tilde{\nu}(x),
$$

where $\tilde{\nu}$ is any smooth extension of $\nu$. We use both of these conventions interchangeably. If we use the latter one, then $N_{x} \Sigma \subset \operatorname{ker} B(x)$ and $\operatorname{Im} B(x) \subset G_{x} \Sigma$. The operator is symmetric and its eigenvalues and corresponding eigenspaces on $G_{x} \Sigma$ are called principal values and principal directions. The mean curvature $H(x)$ of $\Sigma$ associated with the orientation at $x$ is now defined as the trace of $B(x)$ or equivalently the sum of principal values on $G_{x} \Sigma$. Again, the maps $B$ : $\Sigma \rightarrow \mathcal{L}\left(\mathbb{R}^{n} ; \mathbb{R}^{n}\right)$ and $H: \Sigma \rightarrow \mathbb{R}$ are smooth. If $H$ is constant, then $\Sigma$ is said to be critical. The Frobenius norm $|B|$ on $\Sigma$ does not depend on the used orientation. We define also the mean curvature vector field $\mathbf{H}: \Sigma \rightarrow \mathbb{R}^{n}$ by setting $\mathbf{H}=-H \nu$. Notice that $\mathbf{H}$ is independent of the 
choice of orientation. Since $\Sigma$ is compact, then the divergence theorem for hypersurfaces says that for any $f \in C^{1}\left(\mathbb{T}^{n}, \mathbb{R}^{n}\right)$

$$
\int_{\Sigma} \operatorname{div}_{\tau} f \mathrm{~d} \mathcal{H}^{n-1}=-\int_{\Sigma}\langle f, \mathbf{H}\rangle \mathrm{d} \mathcal{H}^{n-1}=\int_{\Sigma} H\langle f, \nu\rangle \mathrm{d} \mathcal{H}^{n-1} .
$$

If $f(x) \in G_{x} \Sigma$ for every $x \in \Sigma$, then the previous formula yields the integration by parts formula

$$
\int_{\Sigma} \varphi \operatorname{div}_{\tau} f \mathrm{~d} \mathcal{H}^{n-1}=-\int_{\Sigma}\left\langle\nabla_{\tau} \varphi, f\right\rangle \mathrm{d} \mathcal{H}^{n-1}
$$

for every $\varphi \in C^{1}(\Sigma)$, in particular $\int_{\Sigma} \operatorname{div}_{\tau} f \mathrm{~d} \mathcal{H}^{n-1}=0$.

While the concept of tangential derivative can be defined similarly on compact $C^{1}$-hypersurfaces, the classical notion of mean curvature is not generally possible for such surfaces due to lack of regularity. However, the following generalization is possible. We say that a compact $C^{1}$-hypersurface $\Gamma \subset \mathbb{T}^{n}$ with an orientation $\nu$ has mean curvature in weak sense, if there exists a Borel function $h \in L^{1}(\Gamma)$ such that for every $f \in C^{\infty}\left(\mathbb{T}^{n} ; \mathbb{R}^{n}\right)$

$$
\int_{\Gamma} \operatorname{div}_{\tau} f \mathrm{~d} \mathcal{H}^{n-1}=\int_{\Gamma} h\langle f, \nu\rangle \mathrm{d} \mathcal{H}^{n-1}
$$

In such a case $h$ is called a weak or distributional mean curvature of $\Gamma$. Moreover, if $h$ is constant, then $\Gamma$ is called stationary. By writing $\Gamma$ locally as a graph surface in an orthonormal coordinates and applying the elliptic regularity results [4, Proposition 7.56] and [12, Theorem 9.19] we obtain the following well-known result.

\section{Lemma 2.1.}

Let $\Gamma \subset \mathbb{T}^{n}$ be a compact $C^{1, \alpha}$-hypersurface with $0<\alpha<1$. Then $\Gamma$ is smooth and critical if and only if it is stationary.

Vector fields, tensors and covariant derivatives on hypersurfaces. Since now for every $\varphi \in$ $C^{\infty}(\Sigma)$ there is a smooth extension $\tilde{\varphi}$ to some open neighborhood of $\Sigma$ and tangential differential is independent of the way an ambient function is extended beyond $\Sigma$, we may define a tangential gradient $\nabla_{\tau} \varphi(x)$ of $\varphi$ at $x$ by setting $\nabla_{\tau} \varphi(x)=\nabla_{\tau} \tilde{\varphi}(x)$. Let $T_{x} \Sigma$ be the tangent space of $\Sigma$ at $x$. Then for every $v \in T_{x} \Sigma$

$$
v(\varphi)=\left\langle z_{v}, \nabla_{\tau} \varphi(x)\right\rangle
$$

with some unique $z_{v} \in G_{x} \Sigma$. Thus the geometric tangent space $G_{x} \Sigma$ can be canonically identified with the tangent space $T_{x} \Sigma$ and, from now on, we just use the notation $T_{x} \Sigma$. The tangent bundle $T \Sigma$ is then the disjoint union of the tangent spaces $T_{x} \Sigma$ equipped with the corresponding smooth structure. Further, we may canonically identify the set of smooth vector fields $\mathfrak{X}(\Sigma)$ on $\Sigma$, that is, the smooth sections $\Sigma \rightarrow T \Sigma$, with the collection

$$
\mathfrak{X}(\Sigma)=\left\{X \in C^{\infty}\left(\Sigma ; \mathbb{R}^{n}\right): X(x) \in T_{x} \Sigma \text { for every } x \in \Sigma\right\},
$$

and for $X \in \mathfrak{X}(\Sigma)$ its action on $\varphi \in C^{\infty}(\Sigma)$ can be seen as $X \varphi=\left\langle X, \nabla_{\tau} \varphi\right\rangle$ on $\Sigma$. As usual, for $X, Y \in \mathfrak{X}(\Sigma)$ the vector field $X Y$ is determined by the rule $X Y \varphi=X(Y \varphi)$.

The Riemannian metric $g$ on $\Sigma$ is the naturally induced flat metric. Keeping the previous identifications in mind this is just the restriction of the standard Euclidean inner product to the hyperspace $T_{x} \Sigma$. The usual flat and sharp operations induced by $g$ for smooth vector and covector fields on $\Sigma$ are denoted by $b$ and $\sharp$ correspondingly. Then for $\varphi \in C^{\infty}(\Sigma)$ the gradient vector field is $\operatorname{grad} \varphi=d \varphi^{\sharp}=\nabla_{\tau} \varphi$.

Slightly abusing the notations we define for a vector field $X \in \mathfrak{X}(\Sigma)$ the (geometric) tangential differential $\mathrm{D}_{\tau} X(x)$ at $x$ as the linear map $\mathbb{R}^{n} \rightarrow \mathbb{R}^{n}$ (or equivalently $T_{x} \Sigma \rightarrow T_{x} \Sigma$ ) by setting

$$
\mathrm{D}_{\tau} X(x)=P_{\Sigma}(x) \mathrm{D}_{\tau} \tilde{X}(x)
$$

where $\tilde{X}$ is any smooth extension of $X$ beyond $\Sigma$. The tangential divergence $\operatorname{div}_{\tau} X(x)$ of $X$ at $x$ is the trace of previous operator and $\operatorname{div}_{\tau} X(x)=\operatorname{div}_{\tau} \tilde{X}(x)$. Now the mappings $\Sigma \rightarrow \mathcal{L}\left(\mathbb{R}^{n} ; \mathbb{R}^{n}\right)$, $x \mapsto \mathrm{D}_{\tau} X(x)$ and $\Sigma \rightarrow \mathbb{R}, x \mapsto \operatorname{div}_{\tau} X(x)$, are smooth. Again, for any $\varphi \in C^{\infty}(\Sigma)$ the tangential Hessian is given by $\mathrm{D}_{\tau}^{2} \varphi=\mathrm{D}_{\tau} \nabla_{\tau} \varphi$, which is a symmetric operator. Further, the Laplace-Beltrami operator or the tangential Laplacian $\Delta_{\tau}$ (of $\Sigma$ ) acting on $\varphi$ can be seen as $\Delta_{\tau} \varphi=\operatorname{tr} \mathrm{D}_{\tau}^{2} \varphi=$ $\operatorname{div}_{\tau} \nabla_{\tau} \varphi$.

The compatible Riemannian connection on $\Sigma$ is the tangential connection $\nabla^{\top}: \mathfrak{X}(\Sigma) \times \mathfrak{X}(\Sigma) \rightarrow$ $\mathfrak{X}(\Sigma)$ given by the rule

$$
\nabla_{X}^{\top} Y=\left(\mathrm{D}_{\tau} Y\right) X
$$


Recall that this is a symmetric connection, i.e., $\nabla_{X}^{\top} Y-\nabla_{Y}^{\top} X=[X, Y]$, where $[X, Y]=X Y-Y X$ is the corresponding commutator.

As usual, for every $m \in \mathbb{N} \cup\{0\}$ and $x \in \Sigma$ we denote the space of $m$-multilinear mappings or $m$-covariant tensors $T_{x} \Sigma \times \cdots \times T_{x} \Sigma \rightarrow \mathbb{R}$ by $T_{0}^{m}\left(T_{x} \Sigma\right)$. Recall the special cases $T_{0}^{1}\left(T_{x} \Sigma\right)=\left(T_{x} \Sigma\right)^{*}$ and $T_{0}^{0}\left(T_{x} \Sigma\right)=\mathbb{R}$. If $m>0$, for given $L, G \in T_{0}^{m}\left(T_{x} \Sigma\right)$ the inner product is given by

$$
\langle L, G\rangle=\sum_{i_{1} \ldots i_{m}} L\left(v_{i_{1}}, \ldots, v_{i_{m}}\right) G\left(v_{i_{1}}, \ldots, v_{i_{m}}\right),
$$

where $v_{1}, \ldots, v_{n-1}$ is any orthonormal basis of $T_{x} \Sigma$. Thus the corresponding tensor norm for $L \in$ $T_{0}^{m}\left(T_{x} \Sigma\right)$ is $|L|=\langle L, L\rangle^{\frac{1}{2}}$. If $L \in T_{0}^{2}\left(T_{x} \Sigma\right)$, then with help of any orthonormal basis $v_{1}, \ldots, v_{n-1}$ of $T_{x} \Sigma$ we may define the trace of $L$ by setting

$$
\operatorname{tr} L=\sum_{i} L\left(v_{i}, v_{i}\right)
$$

The $m$-covariant tensor bundle $T_{0}^{m}(\Sigma)$ is defined by similar means as $T \Sigma$. A covariant $m$ tensor field or section $T: \Sigma \rightarrow T_{0}^{m}(\Sigma)$ is smooth exactly when $T\left(X_{1}, \ldots, X_{m}\right) \in C^{\infty}(\Sigma)$ for every $X_{1}, \ldots, X_{m} \in \mathfrak{X}(\Sigma)$. We denote them by $\mathcal{T}^{m}(\Sigma)$. Recall that $\mathcal{T}^{1}(\Sigma)$ is the collection of the smooth covector fields or 1-forms and $\mathcal{T}^{0}(\Sigma)=C^{\infty}(\Sigma)$.

Now for any (smooth) covariant $m$-tensor field $T \in \mathcal{T}^{m}(\Sigma)$ the covariant derivative of $T$, denoted by $\nabla_{\mathrm{co}} T$, is defined as the element of $\mathcal{T}^{m+1}(\Sigma)$ for which

$$
\nabla_{\mathrm{co}} T\left(X_{1}, \ldots, X_{m}, X_{m+1}\right)=X_{m+1} T\left(X_{1}, \ldots, X_{m}\right)-\sum_{k=1}^{m} T\left(X_{1}, \ldots, \nabla_{X_{m+1}}^{\top} X_{k}, \ldots, X_{m}\right) .
$$

For $\varphi \in C^{\infty}(\Sigma)$ this simply means that $\nabla_{\mathrm{co}} \varphi=d \varphi$ and again for $T \in \mathcal{T}^{m}(\Sigma)$ the $k+1$-th covariant derivative is defined recursively by setting $\nabla_{\mathrm{co}}^{k+1} T=\nabla_{\mathrm{co}}\left(\nabla_{\mathrm{co}}^{k} T\right)$. It is straightforward to compute that for $\varphi \in C^{\infty}(\Sigma)$ the covariant Hessian $\nabla_{\mathrm{co}}^{2} \varphi$ is now the symmetric 2-tensor field obtained by

$$
\nabla_{\mathrm{co}}^{2} \varphi(X, Y)=\left\langle X, \mathrm{D}_{\tau}^{2} \varphi Y\right\rangle \text { for every } X, Y \in \mathfrak{X}(\Sigma),
$$

$\Delta_{\text {со }} \varphi:=\operatorname{tr} \nabla_{\text {со }}^{2} \varphi=\Delta_{\tau} \varphi$ and $\left|\nabla_{\text {со }}^{2} \varphi\right|=\left|\mathrm{D}_{\tau}^{2} \varphi\right|$, where $\left|\mathrm{D}_{\tau}^{2} \varphi\right|$ is the standard Frobenius norm of $\mathrm{D}_{\tau}^{2} \varphi$.

For any $T \in \mathcal{T}^{m}(\Sigma)$ the $C^{k}$-norm on $\Sigma$ is given in the obvious way

$$
\|T\|_{C^{k}(\Sigma)}=\sum_{i=0}^{m} \sup _{\Sigma}\left|\nabla_{\mathrm{co}}^{i} T\right|
$$

and further we set $\|X\|_{C^{k}(\Sigma)}=\left\|X^{b}\right\|_{C^{k}(\Sigma)}$ for every $X \in \mathfrak{X}(\Sigma)$.

Sobolev and Hölder spaces on hypersurfaces. Recall that the Riemannian measure on $\Sigma$ induced by the flat metric $g$ is the restriction of the $n$-1-dimensional Hausdorff measure $\mathcal{H}^{n-1}$ to $\Sigma$. Then the corresponding $p$-Lebesgue space is $L^{p}\left(\Sigma, \mathcal{H}^{n-1}\right)$ for any $1 \leq p \leq \infty$. For every smooth covariant tensor field $T$ on $\Sigma$ the $L^{p}$-norm is now given by

$$
\|T\|_{L^{p}(\Sigma)}=\left(\int_{\Sigma}|T|^{p} \mathrm{~d} \mathcal{H}^{n-1}\right)^{\frac{1}{p}} .
$$

For a $\varphi \in C^{\infty}(\Sigma)$ the Sobolev $W^{k, p}$-norm with $k \in \mathbb{N} \cup\{0\}$ and $1 \leq p \leq \infty$ is given as usual

$$
\|\varphi\|_{W^{k, p}(\Sigma)}=\left(\sum_{j=0}^{k}\left\|\nabla_{\mathrm{co}}^{j} \varphi\right\|_{L^{p}(\Sigma)}^{p}\right)^{\frac{1}{p}} .
$$

Here $\nabla_{\mathrm{co}}^{0} \varphi=\varphi$. For any $1 \leq p<\infty$ the space $W^{k, p}(\Sigma)$ is now the norm completion of $C^{\infty}(\Sigma)$, where any of its element $\varphi$ is considered as the $k+1$-tuple $\left(\varphi, \nabla_{\mathrm{co}}^{1} \varphi, \ldots, \nabla_{\mathrm{co}}^{k} \varphi\right)$. Again, we use the conventional notation $H^{k}(\Sigma)$ for a Hilbert space $W^{k, 2}(\Sigma)$, where the inner product is given in the obvious way

$$
\left\langle\varphi_{1}, \varphi_{2}\right\rangle_{H^{k}}=\sum_{j=0}^{k} \int_{\Sigma}\left\langle\nabla_{\mathrm{co}}^{j} \varphi_{1}, \nabla_{\mathrm{co}}^{j} \varphi_{2}\right\rangle \mathrm{d} \mathcal{H}^{n-1} .
$$

We have the standard Sobolev interpolation for $L^{p}$-norms of covariant derivatives of smooth maps, see [6, Theorem 3.70]. From now on, we denote the space of $C^{\infty}(\Sigma)$-maps with vanishing integrals by $\tilde{C}^{\infty}(\Sigma)$. 
Lemma 2.2 (Basic interpolation).

Let $1 \leq r, q<\infty, 1 \leq p \leq \infty$ and integers $0 \leq j<m$ be selected such that

$$
\frac{1}{p}=\frac{j}{n-1}+\left(\frac{1}{r}-\frac{m}{n-1}\right) \alpha+\frac{1-\alpha}{q},
$$

where $\frac{j}{m} \leq \alpha \leq 1$ and the condition $r=\frac{n-1}{m-j} \neq 1=\alpha$ does not hold. Then there exists a constant $C$ depending on the previous numbers and $\Sigma$ such that for every $\varphi \in C^{\infty}(\Sigma)$

$$
\left\|\nabla_{\mathrm{co}}^{j} \varphi\right\|_{L^{p}(\Sigma)} \leq C\left(\left\|\nabla_{\mathrm{co}}^{m} \varphi\right\|_{L^{r}(\Sigma)}^{\alpha}\|\varphi\|_{L^{q}(\Sigma)}^{1-\alpha}+\beta\|\varphi\|_{L^{1}(\Sigma)}\right) .
$$

Here $\beta=0$, if (i) $j \geq 1$ or (ii) $\Sigma$ is connected and $\varphi \in \tilde{C}^{\infty}(\Sigma)$. Otherwise, $\beta=1$.

\section{Remark 2.3.}

It follows from the previous theorem that in the case $2 \leq n \leq 4$ there is a constant $C$ depending on $\Sigma$ such that $\|\varphi\|_{L^{p}(\Sigma)} \leq C\|\varphi\|_{H^{1}(\Sigma)}$ and $\|\varphi\|_{C^{1}(\Sigma)},\|\varphi\|_{W^{2, p}(\Sigma)} \leq C\|\varphi\|_{H^{3}(\Sigma)}$ for every $1 \leq p \leq 6$ and $\varphi \in C^{\infty}(\Sigma)$. 12.1].

We also recall the following interpolation inequality for covariant tensor fields, see [13, Theorem

Lemma 2.4 (Second order tensor interpolation).

Let $N$ be the number of connected components of $\Sigma$ and suppose that

$$
\frac{1}{p}=\frac{1}{q}+\frac{1}{r}
$$

for $1 \leq p, q, r \leq \infty$. Then for every smooth covariant $k$-tensor field $T$ on $\Sigma$ it holds

$$
\left\|\nabla_{\mathrm{co}} T\right\|_{L^{2 p}(\Sigma)}^{2} \leq N(2 p-2+n-1)\left\|\nabla_{\mathrm{co}}^{2} T\right\|_{L^{r}(\Sigma)}\|T\|_{L^{q}(\Sigma)} \text {. }
$$

Moreover, we need the following estimates, see [10, Lemma 2.3 and Remark 2.4].

Lemma 2.5.

There are constants $C_{n}$, depending only on $n$, and $C_{\Sigma}$, depending on $\Sigma$, such that for every $\varphi \in C^{\infty}(\Sigma)$

$$
\begin{aligned}
\left\|\nabla_{\mathrm{co}}^{2} \varphi\right\|_{L^{2}(\Sigma)}^{2} & \leq\left\|\Delta_{\mathrm{co}} \varphi\right\|_{L^{2}(\Sigma)}^{2}+C_{n} \int_{\Sigma}|B|^{2}\left|\nabla_{\mathrm{co}} \varphi\right|^{2} \mathrm{~d} \mathcal{H}^{n-1} \\
\left\|\nabla_{\mathrm{co}}^{3} \varphi\right\|_{L^{2}(\Sigma)}^{2} & \leq\left\|\nabla_{\mathrm{co}}\left(\Delta_{\mathrm{co}} \varphi\right)\right\|_{L^{2}(\Sigma)}^{2}+C_{\Sigma}\|\varphi\|_{H^{2}(\Sigma)}^{2} .
\end{aligned}
$$

For a continuous map $f: \Sigma \rightarrow \mathbb{R}$ and $0<\alpha<1$ the $C^{\alpha}(\Sigma)$-Hölder semi-norm is given by

$$
[f]_{C^{\alpha}(\Sigma)}=\max _{i=1, \ldots, N} \sup _{\substack{x, y \in \Sigma_{i} \\ x \neq y}} \frac{|f(x)-f(y)|}{d_{g}(x, y)^{\alpha}}
$$

where $d_{g}$ is the length metric induced by $g$. For every $\varphi \in C^{\infty}(\Sigma)$ we define $C^{1, \alpha}(\Sigma)$-norm by setting

$$
\|f\|_{C^{1, \alpha}(\Sigma)}=\|\varphi\|_{C^{1}(\Sigma)}+\sup _{\substack{X \in \mathfrak{X}(\Sigma) \\\|X\|_{C^{1}(\Sigma)} \leq 1}}\left[\nabla_{\operatorname{co}} \varphi(X)\right]_{C^{\alpha}(\Sigma)}
$$

and set the space $C^{1, \alpha}(\Sigma)$ to be the norm completion of the set of $C^{\infty}(\Sigma)$-maps with finite $C^{1, \alpha}(\Sigma)$ norm. Then $C^{1, \alpha}(\Sigma)$ is the space of continuous maps on $\Sigma$ with $C^{1, \alpha}$-extension to some open neighborhood of $\Sigma$. Note that there is several equivalent ways to define $C^{1, \alpha}(\Sigma)$-norm.

The higher order Hölder spaces are defined similarly, but we do not need them. By using the Rellich-Kondarchov theorem, see [14, Thm 2.9], and Lemma 2.2 one obtains the following embedding result.

\section{Lemma 2.6.}

Suppose that $k \geq 3$ is an integer and $1<p$ is a real number. If for given exponent $0<\alpha<1$ the condition

$$
\alpha<2-\frac{n-1}{p}
$$

is true, then the embedding $W^{3, p}(\Sigma) \subset C^{1, \alpha}(\Sigma)$ is compact.

In particular, Lemma 2.6 says that for $n \leq 4$ and $0<\alpha<\frac{1}{2}$ the embedding $H^{3}(\Sigma) \subset C^{1, \alpha}(\Sigma)$ is compact. 
Smooth sets. An open set $E \subset \mathbb{T}^{n}$, with $\operatorname{int}(\bar{E})=E$, is called a smooth set, if the boundary $\partial E$ is a smooth hypersurface. Then $\partial E$ is always a smooth and compact hypersurfaces in $\mathbb{T}^{n}$ so the previous results can be applied on $\partial E$. For the boundary $\partial E$ we always use the natural inside-out orientation denoted by $\nu_{E}$. The classical divergence theorem takes now the following form in $\mathbb{T}^{n}$. For any Lipschitz function $f: \mathbb{T}^{n} \rightarrow \mathbb{R}^{n}$

$$
\int_{E} \operatorname{div} f \mathrm{~d} \mathcal{H}^{n}=\int_{\partial E}\left\langle f, \nu_{E}\right\rangle \mathrm{d} \mathcal{H}^{n-1} .
$$

Further, we denote by $B_{E}$ the second fundamental form on $\partial E$ associated with $\nu_{E}$ and by $H_{E}$ the corresponding boundary mean curvature. Then $E$ is said to be critical, if $\partial E$ is critical, i.e, $H_{E}$ is constant. We use also the shorthand notation $|E|$ for the volume $\mathcal{H}^{n}(E)$. We recall that there exists a regular neighborhood of $\partial E$ say $U_{E}$ such that the signed distance function $\bar{d}_{E}: \mathbb{T}^{n} \rightarrow \mathbb{R}$

$$
\bar{d}_{E}(x)= \begin{cases}\operatorname{dist}(x, \partial E), & x \in \mathbb{T}^{n} \backslash E \\ -\operatorname{dist}(x, \partial E), & x \in E\end{cases}
$$

and the projection mapping $\pi_{\partial E}$ onto $\partial E$ are smooth on $\bar{U}_{E}$ (in particular the latter is well-defined). Again, we may write $\nu_{E}=\nabla \bar{d}_{E}$ and $B_{E}=\mathrm{D}^{2} \bar{d}_{E}$ on $\partial E$. The $C^{k, \alpha}$-sets are defined similarly.

For any $C^{k}$-sets $E$ and $E^{\prime}$ in $\mathbb{T}^{n}$ the $C^{k}$ - "distance" is given by

$$
\left\|E, E^{\prime}\right\|_{C^{k}}=\inf \left\{\|\Phi-\mathrm{id}\|_{C^{k}\left(\mathbb{T}^{n} ; \mathbb{T}^{n}\right)}: \Phi \text { is a } C^{k} \text {-diffeomorphism with } \Phi(E)=E^{\prime}\right\} .
$$

For a smooth set $E$ and an open set $E^{\prime}$ we denote $E^{\prime}=E_{\psi}$ for some $\psi \in C(\partial E)$, if we may write $\partial E^{\prime}$ as a graph of $\psi$ in normal direction over $\partial E$, that is,

$$
\partial E^{\prime}=\left\{x+\psi(x) \nu_{E}(x): x \in \partial E\right\}
$$

and $x+(s+\psi(x)) \nu_{E}(x) \in \mathbb{T}^{n} \backslash E^{\prime}$ with a small positive $s$ for every $x \in \partial E$. Now small $C^{1}$ distance between $E$ and a $C^{k, \alpha}$-set $E^{\prime}$ is equivalent to the graph representation $E^{\prime}=E_{\psi}$ with a $C^{k, \alpha}(\partial E)$-map $\psi$ having a small $C^{1}$-norm.

\section{Lemma 2.7.}

Let $E \subset \mathbb{T}^{n}$ be a smooth set. There exist positive constants $C \geq 1$ and $\delta$ depending on $E$ such that the set $\left\{y \in \mathbb{T}^{n}:\left|\bar{d}_{E}(y)\right| \leq \delta\right\}$ belongs to a regular neighborhood of $\partial E$ and the following hold for any $k \in \mathbb{N} \cup\{\infty\}$ and $0 \leq \alpha<1$.

(i) For any $\psi \in C^{k, \alpha}(\partial E)$ with $\|\psi\|_{C^{1}(\partial E)} \leq \delta$ the set $E_{\psi}$ is defined as a $C^{k, \alpha}$-set, the map $\Phi_{\psi}: \partial E \rightarrow \partial E_{\psi}$, given by

$$
\Phi_{\psi}(x)=x+\psi(x) \nu_{E}(x),
$$

is $C^{k, \alpha}$-diffeomorphism and $\left\|E, E_{\psi}\right\|_{C^{1}} \leq C\|\psi\|_{C^{1}(\partial E)}$.

(ii) If $E^{\prime} \subset \mathbb{T}^{n}$ is a $C^{k, \alpha}$-set with $\left\|E, E^{\prime}\right\|_{C^{1}} \leq \delta$, then there is a unique map $\psi \in C^{k, \alpha}(\partial E)$ for which $E^{\prime}=E_{\psi}$ and $\|\psi\|_{C^{1}(\partial E)} \leq C\left\|E, E^{\prime}\right\|_{C^{1}}$. Moreover, if $\left(E_{t}^{\prime}\right)_{t}$ is a smooth flow (see Definition 2.11) in $\mathbb{T}^{n}$, with $\left\|E, E_{t}^{\prime}\right\|_{C^{1}} \leq \delta$, then the maps $\psi_{t}$, with $E_{t}^{\prime}=E_{\psi_{t}}$, are smoothly parametrized on $\partial E$.

When for $\psi \in C^{\infty}(\partial E)$ its $C^{1}$-norm is small enough, we may control $B_{E_{\psi}}$ and represent $H_{E_{\psi}}$ via $\Phi_{\psi}$ on $\partial E$ in the following way.

\section{Lemma 2.8.}

Let $E \subset \mathbb{T}^{n}$ be a smooth set. There are constants $\delta=\delta(E)$ and $C=C(E)$ and smooth maps

$$
\begin{aligned}
& A: \partial E \times[-\delta, \delta] \times[-\delta, \delta]^{n} \rightarrow T_{0}^{2}(\partial E), \\
& Z: \partial E \times[-\delta, \delta] \times[-\delta, \delta]^{n} \rightarrow T(\partial E) \text { and } \\
& P: \partial E \times[-\delta, \delta] \times[-\delta, \delta]^{n} \rightarrow \mathbb{R}
\end{aligned}
$$

depending on $E$ such that $A(\cdot, t, z)$ and $Z(\cdot, t, z)$ are sections for every pair $(t, z) \in[-\delta, \delta] \times$ $[-\delta, \delta]^{n}, A(\cdot, 0,0)=0$ and the following hold. If $\psi \in C^{\infty}(\partial E)$ and $\|\psi\|_{C^{1}(\partial E)} \leq \delta$, then

$$
\begin{aligned}
H_{E_{\psi}} \circ \Phi_{\psi} & =-\Delta_{\tau} \psi+\left\langle A\left(\cdot, \psi, \nabla_{\tau} \psi\right), \nabla_{\text {co }}^{2} \psi\right\rangle \\
& +\nabla_{\mathrm{co}} \psi\left(Z\left(\cdot, \psi, \nabla_{\tau} \psi\right)\right)+\psi P\left(\cdot, \psi, \nabla_{\tau} \psi\right)+H_{E} \quad \text { and } \\
\left|B_{E_{\psi}} \circ \Phi_{\psi}\right| & \leq C\left(1+\left|D_{\tau}^{2} \psi\right|\right)
\end{aligned}
$$

on $\partial E$. 
Moreover, with the same $\delta$ and $C$ we may assume that the following holds. If $\psi \in C^{\infty}(\partial E)$ with $\|\psi\|_{C^{1}(\partial E)} \leq \delta$, then for every $1 \leq p<\infty, h \in L^{p}\left(\partial E_{\psi}\right)$ and $\varphi \in C^{\infty}\left(\partial E_{\psi}\right)$

$$
\begin{gathered}
C^{-1}\left\|h \circ \Phi_{\psi}\right\|_{L^{p}(\partial E)} \leq\|h\|_{L^{p}\left(\partial E_{\psi}\right)} \leq C\left\|h \circ \Phi_{\psi}\right\|_{L^{p}(\partial E)} \quad \text { and } \\
C^{-1}\left\|\nabla_{\tau}\left(\varphi \circ \Phi_{\psi}\right)\right\|_{L^{p}(\partial E)} \leq\left\|\nabla_{\tau} \varphi\right\|_{L^{p}\left(\partial E_{\psi}\right)} \leq C\left\|\nabla_{\tau}\left(\varphi \circ \Phi_{\psi}\right)\right\|_{L^{p}(\partial E)} .
\end{gathered}
$$

Finally we need the following uniform estimates for low dimensions. The first one says that the first estimate of Remark 2.3 holds with a uniform constant when slightly varying a reference boundary in $C^{1}$-sense.

\section{Lemma 2.9.}

Let $E \subset \mathbb{T}^{n}, n=3,4$, be a smooth set. There exist positive constants $\delta$ and $C$ depending on $E$ such that if $\psi \in C^{\infty}(\partial E)$ with $\|\psi\|_{C^{1}(\partial E)} \leq \delta$, then for every $1 \leq p \leq 6$ and $\varphi \in C^{\infty}\left(\partial E_{\psi}\right)$

$$
\|\varphi\|_{L^{p}\left(\partial E_{\psi}\right)} \leq C\|\varphi\|_{H^{1}\left(\partial E_{\psi}\right)} .
$$

The second one says that we can control uniformly $L^{p}$-norm of $\nabla_{\tau} \varphi$ (up to $p \leq 6$ ) by the $L^{2}$-norm of $\Delta_{\tau} \varphi$ and the $H^{1}$-norm of $\varphi$ when slightly varying a reference boundary in $H^{3}$-sense.

Lemma 2.10.

Let $E \subset \mathbb{T}^{n}, n=3,4$, be a smooth set. There are constants $\delta$ and $C$ depending on $E$ such that if $\psi \in C^{\infty}(\partial E)$ and $\|\psi\|_{C^{1}(\partial E)} \leq \delta$, then for every $1 \leq p \leq 6$ and $\varphi \in C^{\infty}\left(\partial E_{\psi}\right)$

$$
\left\|\nabla_{\tau} \varphi\right\|_{L^{p}\left(\partial E_{\psi}\right)} \leq C\left(\left\|\Delta_{\tau} \varphi\right\|_{L^{2}\left(\partial E_{\psi}\right)}+\|\varphi\|_{H^{1}\left(\partial E_{\psi}\right)}\right) .
$$

Lemma 2.9 and Lemma 2.10 hold also in the case $n>4$, if we replace the upper bound 6 with smaller number depending on $n$. This number converges to 2 by above as $n$ tends to infinity. We will prove these results except Lemma 2.7 in Appendix.

Volume preserving mean curvature flow and strictly stable sets. Let us first give the formal definition of smooth flow in this setting.

\section{Definition 2.11 (Smooth flow).}

The parametrized family $\left(E_{t}\right)_{t \in[0, T[}$ of smooth sets in $\mathbb{T}^{n}$ with $0<T \leq \infty$ is a smooth flow with an initial set or initial datum $E_{0}$, if there exists a smooth map $\Phi: \mathbb{T}^{n} \times\left[0, T\left[\rightarrow \mathbb{T}^{n}\right.\right.$ such that $\Phi_{0}=\mathrm{id}_{\mathbb{T}^{n}}, \Phi_{t}:=\Phi(\cdot, t)$ is a (smooth) diffeomorphism and $E_{t}=\Phi_{t}\left(E_{0}\right)$ for every $t \in[0, T[$. Again, for every $0 \leq t<T$, the (outer) normal velocity of the flow on $\partial E_{t}$ at the time $t$ is defined by setting

$$
V_{t}=\left\langle\left.\partial_{s} \Phi_{s+t}\right|_{s=0} \circ \Phi_{t}^{-1}, \nu_{E_{t}}\right\rangle
$$

The normal velocity $V_{0}$ on $\partial E_{0}$ is called an initial velocity. If we do not emphasize the time interval [0,T [, we write $\left(E_{t}\right)_{t}$ for short. Also, for the unit normal field $\nu_{E_{t}}$ the corresponding second fundamental form $B_{E_{t}}$ and the boundary mean curvature $H_{E_{t}}$ we use the shorthand notations $\nu_{t}$, $B_{t}$ and $H_{t}$, when there is no possibility of confusion. In the previous definition $\Phi$ is called a smoothly parametrized family of diffeomorphisms and we may suggestively denote it by $\left(\Phi_{t}\right)_{t \in[0, T[}$ or $\left(\Phi_{t}\right)_{t}$. The normal velocity $V_{t}$ on $\partial E_{t}$ does not depend on the choice of the parametrization $\Phi$. Recall the first variation of volume under the flow

$$
\frac{\mathrm{d}}{\mathrm{d} t}\left|E_{t}\right|=\int_{\partial E_{t}} V_{t} \mathrm{~d} \mathcal{H}^{n-1}
$$

and again the first variation of perimeter

$$
\frac{\mathrm{d}}{\mathrm{d} t}\left|\partial E_{t}\right|=\int_{\partial E_{t}} V_{t} H_{t} \mathrm{~d} \mathcal{H}^{n-1}
$$

We say that $\left(E_{t}\right)_{t}$ is volume preserving, if $\left|E_{t}\right|$ is a constant function in time. According to (2.12) this is possible exactly when $V_{t}$ has a vanishing integral over $\partial E_{t}$ for every $t$. Conversely, one may show that if $E$ is a smooth set in $\mathbb{T}^{n}$ and $\varphi \in \tilde{C}^{\infty}(\partial E)$, there is a smooth volume preserving flow $\left(E_{t}\right)_{t}$ starting from $E$ such that initial velocity on $\partial E$ is $\varphi$. Then, by using a simple approximation argument, it follows from (2.13) that a smooth set $E$ is critical if and only if for every smooth volume preserving flow $\left(E_{t}\right)_{t}$ starting from $E$

$$
\left.\frac{\mathrm{d}}{\mathrm{d} t}\left|\partial E_{t}\right|\right|_{t=0}=0
$$


Further, if in the previous setting the flow admits a parametrization $\Phi$ autonomous with respect to time, i.e., $\partial_{t} \Phi=X(\Phi)$ with a $X \in C^{\infty}\left(\mathbb{T}^{n} ; \mathbb{R}^{n}\right)$, then the second variation of perimeter at $t=0$ is

$$
\left.\frac{\mathrm{d}^{2}}{\mathrm{~d} t^{2}}\left|\partial E_{t}\right|\right|_{t=0}=\int_{\partial E}\left|\nabla_{\tau} V_{0}\right|^{2}-\left|B_{E}\right|^{2} V_{0}^{2} \mathrm{~d} \mathcal{H}^{n-1},
$$

see [2, Remark 3.3]. This motivates us to define for every smooth $E$ in $\mathbb{T}^{n}$ the quadratic form $\partial^{2} P(E): \tilde{H}^{1}(\partial E) \rightarrow \mathbb{R}$, where $\tilde{H}^{1}(\partial E)$ is the space of $H^{1}(\partial E)$-maps with vanishing integral over $\partial E$, by setting first for every $\varphi \in \tilde{C}^{\infty}(\partial E)$

$$
\partial^{2} P(E)[\varphi]=\int_{\partial E}\left|\nabla_{\tau} \varphi\right|^{2}-\left|B_{E}\right|^{2} \varphi^{2} \mathrm{~d} \mathcal{H}^{n-1}
$$

and then extending $\partial^{2} P(E)$ to $\tilde{H}^{1}(\partial E)$ in the obvious way. We say that a smooth and critical set $E$ in $\mathbb{T}^{n}$ is stable, if $\partial^{2} P(E)(\varphi) \geq 0$ for every $\varphi \in \tilde{H}^{1}(\partial E)$.

For every smooth set $E$ in $\mathbb{T}^{n}$ the space of infinitesimal translations on $\partial E$ is given by

$$
T(\partial E)=\left\{\left\langle\nu_{E}, p\right\rangle: p \in \mathbb{R}^{n}\right\} .
$$

Notice that $T(\partial E) \subset \tilde{C}^{\infty}(\partial E)$. These maps correspond to the initial velocities of the linear translations along a vector. It follows from [2, Theorem 3.1] that if $E_{t}=E+t p$, then the second variation at $t=0$ of perimeter under this translation is $\partial^{2} P(E)\left[\left\langle\nu_{E}, p\right\rangle\right]$. Since translations do not change perimeter, then $\partial^{2} P(E)\left[\left\langle\nu_{E}, p\right\rangle\right]=0$. Thus $\partial^{2} P(\partial E)$ is always zero on $T(\partial E)$, which is also easy to compute directly by using the definition. This leads us to define the strictly stable sets in the following way, see [2].

Definition 2.12 (Strictly stable set).

A smooth and critical set $F$ in $\mathbb{T}^{n}$ is strictly stable, if $\partial^{2} P(E)[\varphi]>0$ for every nonzero map $\varphi \in \tilde{H}^{1}(\partial F) \cap T^{\perp, L^{2}}(\partial F)$, where $T^{\perp, L^{2}}(\partial F)$ is the orthogonal complement of $T(\partial F)$ in $L^{2}(\partial F)$.

In the previous definition, we may replace the condition $\partial^{2} P(E)[\varphi]>0$ for every nonzero map $\varphi \in \tilde{H}^{1}(\partial F) \cap T^{\perp, L^{2}}(\partial F)$ with $\partial^{2} P(E)[\varphi]>0$ for every map $\varphi \in \tilde{H}^{1}(\partial F) \backslash T(\partial F)$ due to the fact that $\Delta_{\tau}\left\langle\nu_{F}, p\right\rangle=-\left|B_{F}\right|^{2}\left\langle\nu_{F}, p\right\rangle$ for every $p \in \mathbb{R}^{n}$. As mentioned already in Introduction such sets are always isolated local perimeter minimizers. For a strictly stable set $F$ any critical set with the same volume being close enough to $F$ in $H^{3}$-sense is a translate of $F$.

\section{Lemma 2.13.}

Let $F \subset \mathbb{T}^{n}, n=3,4$, be a strictly stable set. There exists a positive $\delta=\delta(F)$ such that if $F_{\psi}$ is critical, $\left|F_{\psi}\right|=|F|$ and $\|\psi\|_{H^{3}(\partial F)} \leq \delta_{1}$ for $\psi \in C^{\infty}(\partial F)$, then $F_{\psi}$ is a translate of $F$.

This result follows from the proof of [2, Theorem 3.9] (for clarity see the proof of [1, Proposition 2.7] although it concerns only the case $n=3$ ) and Remark 2.3. Moreover, being near to a strictly stable set in $H^{3}$-sense implies that the quadratic form controls $H^{1}$-norm for the $\tilde{C}^{\infty}$-functions orthogonal to the infinitesimal translations in $L^{2}$-sense, see [1, Lemma 2.6].

\section{Lemma 2.14.}

Let $F \subset \mathbb{T}^{n}, n=3,4$, be a strictly stable set. There exist $\sigma_{1}=\sigma_{1}(F)$ and $\delta=\delta(F)$ such that if $\|\psi\|_{H^{3}(\partial F)} \leq \delta_{1}$ for $\psi \in C^{\infty}(\partial F)$, then for every $\varphi \in \tilde{C}^{\infty}\left(\partial F_{\psi}\right) \cap T^{\perp, L^{2}}\left(\partial F_{\psi}\right)$

$$
\sigma_{1}\|\varphi\|_{H^{1}\left(\partial F_{\psi}\right)}^{2} \leq \partial^{2} P\left(F_{\psi}\right)[\varphi]
$$

The authors prove the previous result in the case $n=3$ and $H^{3}$-closeness being replaced by $W^{2, p}$-closeness with any $p>2$, but clearly the same arguments go through for any $n \geq 2$, if we use the condition $p>\max \{n-1,2\}$ instead of $p>2$. Hence the result follows from Remark 2.3.

As already presented in Introduction, a volume preserving mean curvature flow $\left(E_{t}\right)_{t}$ in $\mathbb{T}^{n}$ is a smooth flow obeying the rule

$$
V_{t}=\bar{H}_{t}-H_{t}
$$

where $\bar{H}_{t}$ is the integral average of $H_{t}$ over $\partial E_{t}$. Then by (2.12) and (2.13) we see that

$$
\frac{\mathrm{d}}{\mathrm{d} t}\left|E_{t}\right|=0 \quad \text { and } \quad \frac{\mathrm{d}}{\mathrm{d} t}\left|\partial E_{t}\right|=-\int_{E_{t}}\left(\bar{H}_{t}-H_{t}\right)^{2} \mathrm{~d} \mathcal{H}^{n-1} \leq 0
$$

so the flow is volume preserving and decreases boundary area. The existence and uniqueness of such a flow for a given smooth initial datum is well-known. This is usually known as short time existence. Also, the maximal lifetime of the flow is bounded by below when slightly varying the initial set in the $C^{1, \alpha}$-topology with $\alpha>0$. 
Theorem 2.15 (Short Time Existence).

Let $E \subset \mathbb{T}^{n}$ be a smooth set and $0<\alpha<1$. There are positive constants $\delta$ and $T$ depending on $E$ and $\alpha$ such that if $E_{0}$ is a smooth set in $\mathbb{T}^{n}$ of the form $\partial E_{0}=E_{\psi_{0}}$, where $\psi_{0} \in C^{\infty}(\partial E)$ and $\left\|\psi_{0}\right\|_{C^{1, \alpha}(\partial E)} \leq \delta$, then there exists a unique volume preserving mean curvature flow in $\mathbb{T}^{n}$ with the initial datum $E_{0}$ and the maximal lifetime of flow is at least $T$.

The above result has been proved for bounded smooth sets in $\mathbb{R}^{n}$, see [9, Main Theorem] and clearly it is similar to prove it in the setting of the flat torus $\mathbb{T}^{n}$. From now on, when there is no danger of confusion, we denote a maximal lifetime of a given volume preserving mean curvature flow $\left(E_{t}\right)_{t}$ by $T^{*}$.

\section{3. $L^{2}$-MONOTONICITY}

In this section we prove a monotonicity result, which is the basis of our analysis. It states that if $\left(E_{t}\right)_{t}$ is a volume preserving mean curvature flow with a smooth initial datum near enough to a strictly stable set $F$ in $H^{3}$-sense, then it stays near $F$ in the $H^{3}$-sense for the whole lifespan of the flow, the initial velocity of flow decreases exponentially in $L^{2}$-sense and the quantity $\left\|\nabla_{\tau} H_{t}\right\|_{L^{2}\left(\partial E_{t}\right)}^{2}+C_{0}\left\|\bar{H}_{t}-H_{t}\right\|_{L^{2}\left(\partial E_{t}\right)}^{2}$ is decreasing in time with sufficiently large $C_{0}$. More precisely we have the following result.

Theorem 3.1 (Monotonicity near strictly stable set).

Let $F \subset \mathbb{T}^{n}(n=3,4)$ be a strictly stable set. There are positive constants $C_{0}$ and $\varepsilon_{0}$ depending on $F$ such that for every $0<\varepsilon \leq \varepsilon_{0}$ there is a positive $\gamma_{\varepsilon}<\varepsilon$ such that if $\left(E_{t}\right)_{t}$ is a volume preserving mean curvature flow starting from a smooth set $E_{0}=F_{\psi_{0}}$, where $\psi_{0} \in C^{\infty}(\partial F)$ and $\left\|\psi_{0}\right\|_{H^{3}(\partial F)} \leq \gamma_{\varepsilon}$, then for every $t \in\left[0, T^{*}\left[\right.\right.$ (where $T^{*}>0$ is the maximal lifetime of flow) we may write $E_{t}=F_{\psi_{t}}$ with $\psi_{t} \in C^{\infty}(\partial F),\left\|\psi_{t}\right\|_{H^{3}(\partial F)} \leq \varepsilon$,

$$
\begin{aligned}
\left\|\bar{H}_{t}-H_{t}\right\|_{L^{2}\left(\partial E_{t}\right)}^{2} & \leq\left\|\psi_{0}\right\|_{H^{3}(\partial F)} e^{-\sigma_{1} t} \text { and } \\
\frac{\mathrm{d}}{\mathrm{d} t}\left[\left\|\nabla_{\tau} H_{t}\right\|_{L^{2}\left(\partial E_{t}\right)}^{2}+C_{0}\left\|\bar{H}_{t}-H_{t}\right\|_{L^{2}\left(\partial E_{t}\right)}^{2}\right] & \leq 0,
\end{aligned}
$$

where $\sigma_{1}$ is as in Lemma 2.14 .

\section{Remark 3.2.}

As a byproduct of the proof of Theorem 3.1 we may replace zero with $-\frac{1}{2}\left\|\Delta_{\tau} H_{t}\right\|_{L^{2}\left(\partial E_{t}\right)}^{2}$ on the right-hand side of 3.2. However, we do not need that fact.

Before proving Theorem 3.1 we introduce the following useful geometric quantity employed, for instance, in [1]. For any open set $E \subset \mathbb{T}^{n}$ we define the map $D_{E}$ from the collection of the open subsets of $\mathbb{T}^{n}$ to $\left[0, \infty\left[\right.\right.$ by setting for any open $E^{\prime} \subset \mathbb{T}^{n}$

$$
D_{E}\left(E^{\prime}\right):=\int_{E^{\prime} \Delta E} \operatorname{dist}_{\partial E} \mathrm{~d} \mathcal{H}^{n}=\int_{E^{\prime}} \bar{d}_{E} \mathrm{~d} \mathcal{H}^{n}-\int_{E} \bar{d}_{E} \mathrm{~d} \mathcal{H}^{n} .
$$

In case of any smooth set $E \subset \mathbb{T}^{n}$ this concept of "weak distance" turns out to be very useful in terms of controlling the $L^{2}(\partial E)$-norm of the corresponding function of given $C^{1}$-graph in normal direction over $\partial E$, when the corresponding $C^{1}(\partial F)$-norm is sufficiently small. To observe this, choose $\delta=\delta(E)$ so small that by Lemma 2.7 for any $\psi \in C^{1}(\partial E)$ with $\|\psi\|_{C^{1}(\partial E)} \leq \delta$ the set $E_{\psi}$ is defined as a $C^{1}$-set. Moreover, we may assume that for every $s \in[-\delta, \delta]$ the map $\Phi_{s}=\mathrm{id}+s \nabla \bar{d}_{E}$ is defined as a smooth diffeomorphism from some tubular neighborhood of $\partial E$ to its image. With help of the coarea formula one may compute

$$
D_{E}\left(E_{\psi}\right)=\int_{\left[0,\|\psi\|_{L}(\partial E)\right]} s\left[\int_{\{x \in \partial E: \psi(x)>s\}} J_{\tau} \Phi_{s} \mathrm{~d} \mathcal{H}^{n-1}+\int_{\{x \in \partial E: \psi(x)<-s\}} J_{\tau} \Phi_{-s} \mathrm{~d} \mathcal{H}^{n-1}\right] \mathrm{d} s .
$$

Since now $J_{\tau} \Phi_{s} \rightarrow 1$ uniformly on $\partial E$ as $s \rightarrow 0$, then by decreasing $\delta$, if necessary, it follows from the Cavalieri's principle that there exists $C \geq 1$ depending on $\delta$ such that for every $\psi \in C^{1}(\partial E)$ with $\|\psi\|_{C^{1}(\partial E)} \leq \delta$

$$
C^{-1}\|\psi\|_{L^{2}(\partial E)}^{2} \leq D_{E}\left(E_{\psi}\right) \leq C\|\psi\|_{L^{2}(\partial E)}^{2} .
$$

Again, for any open subset $E \subset \mathbb{T}^{n}$ the map $D_{E}$ behaves well under any smooth flow. If $\left(E_{t}\right)_{t}$ is a smooth flow in $\mathbb{T}^{n}$ with a normal velocity $V_{t}$, then $D_{E}\left(E_{t}\right)$ is differentiable in time and it is straightforward to calculate

$$
\frac{\mathrm{d}}{\mathrm{d} t} D_{E}\left(E_{t}\right)=\int_{\partial E_{t}} \bar{d}_{E} V_{t} \mathrm{~d} \mathcal{H}^{n-1}
$$


Now the importance of this quantity lies on the fact, that for any smooth critical set $F \subset \mathbb{T}^{n}$ and $\psi \in C^{\infty}(\partial F)$ with sufficiently small $C^{1}$-norm, $\left\|\nabla_{\tau} H_{F_{\psi}}\right\|_{L^{2}(\partial F)}$ and $D_{F}\left(F_{\psi}\right)$ together control the $H^{3}$-norm of $\psi$.

\section{Lemma 3.3.}

Let $F \subset \mathbb{T}^{n}$ be a smooth critical set. There are positive constants $K=K(F) \geq 1$ and $\delta=\delta(F)$ such that whenever $\psi \in C^{\infty}(F)$ satisfies $\|\psi\|_{C^{1}(\partial F)} \leq \delta$, then

$$
K^{-1}\left(\left\|\nabla_{\tau} H_{F_{\psi}}\right\|_{L^{2}\left(\partial F_{\psi}\right)}+\sqrt{D_{F}\left(F_{\psi}\right)}\right) \leq\|\psi\|_{H^{3}(\partial F)} \leq K\left(\left\|\nabla_{\tau} H_{F_{\psi}}\right\|_{L^{2}\left(\partial F_{\psi}\right)}+\sqrt{D_{F}\left(F_{\psi}\right)}\right) .
$$

Proof.

We prove only the inequality

$$
\|\psi\|_{H^{3}(\partial F)} \leq K\left(\left\|\nabla_{\tau} H_{F_{\psi}}\right\|_{L^{2}\left(\partial F_{\psi}\right)}+\sqrt{D_{F}\left(F_{\psi}\right)}\right) .
$$

The another one is easier to show. Again, it follows from (2.11) and (3.4), that it suffices to find positive $\delta$ and $K$ such that for every $\psi \in C^{\infty}(\partial F)$ with $\|\psi\|_{C^{1}(\partial E)} \leq \delta$

$$
\|\psi\|_{H^{3}(\partial F)} \leq K\left(\left\|\nabla_{\tau}\left(H_{F_{\psi}} \circ \Phi_{\psi}\right)\right\|_{L^{2}(\partial F)}+\|\psi\|_{L^{2}(\partial F)}\right)
$$

where $\Phi_{\psi}: \partial F \rightarrow \partial F_{\psi}$ is defined as in Lemma 2.7. To this end we will prove the following auxiliary result. For suitable positive constants $\delta^{\prime}$ and $K^{\prime}$ the estimate

$$
\left|\nabla_{\tau}\left(H_{F_{\psi}} \circ \Phi_{\psi}\right)\right|^{2} \geq \frac{\left|\nabla_{\mathrm{co}}\left(\Delta_{\mathrm{co}} \psi\right)\right|^{2}}{2}-\frac{\left|\nabla_{\mathrm{co}}^{3} \psi\right|^{2}}{4}-K^{\prime}\left(|\psi|^{2}+\left|\nabla_{\mathrm{co}} \psi\right|^{2}+\left|\nabla_{\mathrm{co}}^{2} \psi\right|^{2}+\left|\nabla_{\mathrm{co}}^{2} \psi\right|^{4}\right)
$$

holds on $\partial F$ for every $\psi \in C^{\infty}(\partial F)$ with $\|\psi\|_{C^{1}(\partial E)} \leq \delta^{\prime}$. Due to the compactness of $\partial F$ we have only to show this holds locally. Let $\delta^{\prime \prime}$ be the constant for $F$ as in Lemma 2.8. Then for every $\psi \in C^{\infty}(\partial F)$ with $\|\psi\|_{C^{1}(\partial F)} \leq \delta^{\prime \prime}$

$$
\begin{aligned}
H_{F_{\psi}} \circ \Phi_{\psi} & =-\Delta_{\tau} \psi+\left\langle A\left(\cdot, \psi, \nabla_{\tau} \psi\right), \nabla_{\mathrm{co}}^{2} \psi\right\rangle \\
& +\nabla_{\mathrm{co}} \psi\left(Z\left(\cdot, \psi, \nabla_{\tau} \psi\right)\right)+\psi P\left(\cdot, \psi, \nabla_{\tau} \psi\right)+H_{F}
\end{aligned}
$$

where

$$
\begin{aligned}
& A: \partial F \times\left[-\delta^{\prime \prime}, \delta^{\prime \prime}\right] \times\left[-\delta^{\prime \prime}, \delta^{\prime \prime}\right]^{n} \rightarrow T_{0}^{2}(\partial F), \\
& Z: \partial F \times\left[-\delta^{\prime \prime}, \delta^{\prime \prime}\right] \times\left[-\delta^{\prime \prime}, \delta^{\prime \prime}\right]^{n} \rightarrow T(\partial F) \text { and } \\
& P: \partial F \times\left[-\delta^{\prime \prime}, \delta^{\prime \prime}\right] \times\left[-\delta^{\prime \prime}, \delta^{\prime \prime}\right]^{n} \rightarrow \mathbb{R}
\end{aligned}
$$

are smooth maps depending on $F$ and $A(\cdot, 0,0): \partial F \rightarrow T_{0}^{2}(\partial F)$ is the zero tensor field. For a fixed point $x_{0} \in \partial F$ choose a local orthonormal vector frame $\left(E_{1}, \ldots, E_{n-1}\right)$ in some open neighborhood $U_{x_{0}} \subset \partial F$ of $x_{0}$. Then we may write for every $\psi \in C^{\infty}(\partial F)$ with $\|\psi\|_{C^{1}(\partial E)} \leq \delta^{\prime \prime}$

$$
\begin{aligned}
& \left\langle A\left(\cdot, \psi, \nabla_{\tau} \psi\right), \nabla_{\mathrm{co}}^{2} \psi\right\rangle=\sum_{i j} a_{i j}\left(\cdot, \psi, \nabla_{\tau} \psi\right) \nabla_{\mathrm{co}}^{2} \psi\left(E_{i}, E_{j}\right) \quad \text { and } \\
& \nabla_{\mathrm{co}} \psi\left(Z\left(\cdot, \psi, \nabla_{\tau} \psi\right)\right)=\sum_{i} z_{i}\left(\cdot, \psi, \nabla_{\tau} \psi\right) \nabla_{\mathrm{co}} \psi\left(E_{i}\right)
\end{aligned}
$$

where $a_{i j}, z_{i}: U_{x_{0}} \times\left[-\delta^{\prime \prime}, \delta^{\prime \prime}\right] \times\left[-\delta^{\prime \prime}, \delta^{\prime \prime}\right]^{n} \rightarrow \mathbb{R}$ are the smooth functions given by

$$
\begin{aligned}
a_{i j}(x, t, z) & =A(x, t, z)\left(E_{i}(x), E_{j}(x)\right) \text { and } \\
z_{i}(x, t, z) & =\left\langle E_{i}(x), Z(x, t, z)\right\rangle
\end{aligned}
$$

for every $(x, t, z) \in U_{x_{0}} \times\left[-\delta^{\prime \prime}, \delta^{\prime \prime}\right] \times\left[-\delta^{\prime \prime}, \delta^{\prime \prime}\right]^{n}$. Notice that then $\mid\left(a_{i j}(x, t, z)|\leq| A(x, t, z) \mid\right.$ and $\left|z_{i}(x, t, z)\right| \leq|Z(x, t, z)|$. By taking tangential gradient over (3.8) (notice that for every $\varphi \in C^{\infty}(\partial F)$ we may write $\nabla_{\tau} \varphi=\sum_{i}\left(\nabla_{E_{i}} \varphi\right) E_{i}$ in $\left.U_{p_{0}}\right)$ and using the expressions (3.9) and (3.10) 
we obtain

$$
\begin{aligned}
\nabla_{\tau}\left(H_{F_{\psi}} \circ \Phi_{\psi}\right) & =-\nabla_{\tau}\left(\Delta_{\mathrm{co}} \psi\right)+\sum_{i j k} a_{i j}\left(\cdot, \psi, \nabla_{\tau} \psi\right) \nabla_{\mathrm{co}}^{3} \psi\left(E_{i}, E_{j}, E_{k}\right) E_{k} \\
& +\sum_{i j k} a_{i j}\left(\cdot, \psi, \nabla_{\tau} \psi\right)\left(\nabla_{\mathrm{co}}^{2} \psi\left(\nabla_{E_{k}} E_{i}, E_{j}\right)+\nabla_{\mathrm{co}}^{2} \psi\left(E_{i}, \nabla_{E_{k}} E_{j}\right)\right) E_{k} \\
& +\sum_{i j} \nabla_{\mathrm{co}}^{2} \psi\left(E_{i}, E_{j}\right) \nabla_{\tau}\left(a_{i j}\left(\cdot, \psi, \nabla_{\tau} \psi\right)\right) \\
& +\sum_{i k} z_{i}\left(\cdot, \psi, \nabla_{\tau} \psi\right)\left(\nabla_{\mathrm{co}}^{2} \psi\left(E_{i}, E_{k}\right)+\nabla_{\mathrm{co}} \psi\left(\nabla_{E_{k}} E_{i}\right)\right) E_{k} \\
& +\sum_{i} \nabla_{\mathrm{co}} \psi\left(E_{i}\right) \nabla_{\tau}\left(z_{i}\left(\cdot, \psi, \nabla_{\tau} \psi\right)\right) \\
& +P\left(\cdot, \psi, \nabla_{\tau} \psi\right) \nabla_{\tau} \psi+\psi \nabla_{\tau}\left(P\left(\cdot, \psi, \nabla_{\tau} \psi\right)\right)
\end{aligned}
$$

Recall that $\Delta_{\mathrm{co}} \psi=\Delta_{\tau} \psi$ and $H_{F}$ is a constant thus vanishing after taking gradient over (3.8). Again, for any smooth map $u: U_{x_{0}} \times\left[-\delta^{\prime \prime}, \delta^{\prime \prime}\right] \times\left[-\delta^{\prime \prime}, \delta^{\prime \prime}\right]^{n} \rightarrow \mathbb{R}$ one computes

$$
\begin{aligned}
\nabla_{\tau}\left(u\left(\cdot, \psi, \nabla_{\tau} \psi\right)\right) & =\nabla_{\tau} u\left(\cdot, \psi, \nabla_{\tau} \psi\right)+\partial_{t} u\left(\cdot, \psi, \nabla_{\tau} \psi\right) \nabla_{\tau} \psi \\
& +\mathrm{D}_{\tau}^{2} \psi \nabla_{n} u\left(\cdot, \psi, \nabla_{\tau} \psi\right)-\left\langle\nu_{F}, \nabla_{n} u\left(\cdot, \psi, \nabla_{\tau} \psi\right)\right\rangle B_{F} \nabla_{\tau} \psi
\end{aligned}
$$

It follows from $A(\cdot, 0,0)$ being the zero tensor field on $\partial F$ and uniform continuity on compact sets, that there exist $0<\delta^{\prime}<\delta^{\prime \prime}$ and $C \geq 1$ such that for every $(x, t, z) \in \partial F \times\left[-\delta^{\prime}, \delta^{\prime}\right] \times\left[-\delta^{\prime}, \delta^{\prime}\right]^{n}$

$$
\begin{aligned}
|A(x, t, z)| & \leq \frac{1}{64(n-1)^{3}} \quad \text { and } \\
|Z(x, t, z)|,|P(x, t, z)| & \leq C .
\end{aligned}
$$

By shrinking $U_{x_{0}}$ and increasing $C$, if necessary, we may assume that each $\left|\nabla_{E_{k}} E_{i}\right|$ is bounded by $C$ in $U_{x_{0}}$. Since $\left|\nabla_{\tau} \psi\right|=\left|\nabla_{\text {co }} \psi\right|$ and $\left|\mathrm{D}_{\tau}^{2} \psi\right|=\left|\nabla_{\text {co }}^{2} \psi\right|$ on $\partial F$ for every $\psi \in C^{\infty}(\partial F)$, then again by shrinking $U_{x_{0}}$ and increasing $C$, if needed, it follows from (3.12) that for every $\psi \in C^{\infty}(\partial F)$ with $\|\psi\|_{C^{1}(\partial F)} \leq \delta^{\prime}$

$$
\left|\nabla_{\tau}\left(a_{i j}\left(\cdot, \psi, \nabla_{\tau} \psi\right)\right)\right|,\left|\nabla_{\tau}\left(z_{i}\left(\cdot, \psi, \nabla_{\tau} \psi\right)\right)\right|,\left|\nabla_{\tau}\left(P\left(\cdot, \psi, \nabla_{\tau} \psi\right)\right)\right| \leq C\left(1+\left|\nabla_{\mathrm{co}}^{2} \psi\right|\right)
$$

in $U_{x_{0}}$. Recalling expression (3.11) for such $\psi$ we denote

$$
a_{\psi}=\sum_{i j k} a_{i j}\left(\cdot, \psi, \nabla_{\tau} \psi\right) \nabla_{\mathrm{co}}^{3} \psi\left(E_{i}, E_{j}, E_{k}\right) E_{k}
$$

and the sum of the lower order terms by $b_{\psi}$. Thus we may write shortly in $U_{x_{0}}$

$$
\nabla_{\tau}\left(H_{F_{\psi}} \circ \Phi_{\psi}\right)=-\nabla_{\tau}\left(\Delta_{\mathrm{co}} \psi\right)+a_{\psi}+b_{\psi}
$$

Now by using (3.13) we have

$$
\left|a_{\psi}\right| \leq \frac{\left|\nabla_{\mathrm{co}}^{3} \psi\right|}{64}
$$

in $U_{p}$. Again, by applying the estimates (3.14) and (3.15) on $b_{\psi}$ we find a positive constant $C^{\prime}$ depending on $C, \delta^{\prime}$ and $n$ such that in $U_{x_{0}}$

$$
\left|b_{\psi}\right| \leq C^{\prime}\left(|\psi|+\left|\nabla_{\mathrm{co}} \psi\right|+\left|\nabla_{\mathrm{co}}^{2} \psi\right|+\left|\nabla_{\mathrm{co}}^{2} \psi\right|^{2}\right) .
$$

Thus by using (3.16) and (3.17) as well as the Cauchy-Schwarz and Young's inequalities, we have in $U_{x_{0}}$

$$
\begin{aligned}
\left|\nabla_{\tau}\left(H_{F_{\psi}} \circ \Phi_{\psi}\right)\right|^{2} & =\left|-\nabla_{\tau}\left(\Delta_{\mathrm{co}} \psi\right)+a_{\psi}+b_{\psi}\right|^{2} \\
& \geq\left|\nabla_{\tau}\left(\Delta_{\mathrm{co}} \psi\right)\right|^{2}-2\left|\nabla_{\tau}\left(\Delta_{\mathrm{co}} \psi\right)\right|\left|a_{\psi}+b_{\psi}\right| \\
& \geq \frac{1}{2}\left|\nabla_{\tau}\left(\Delta_{\mathrm{co}} \psi\right)\right|^{2}-8\left|a_{\psi}+b_{\psi}\right|^{2} \\
& \geq \frac{1}{2}\left|\nabla_{\tau}\left(\Delta_{\mathrm{co}} \psi\right)\right|^{2}-16\left|a_{\psi}\right|^{2}-16\left|b_{\psi}\right|^{2} \\
& \geq \frac{1}{2}\left|\nabla_{\tau}\left(\Delta_{\mathrm{co}} \psi\right)\right|^{2}-\frac{1}{4}\left|\nabla_{\mathrm{co}}^{3} \psi\right|^{2}-64\left(C^{\prime}\right)^{2}\left(|\psi|^{2}+\left|\nabla_{\mathrm{co}} \psi\right|^{2}+\left|\nabla_{\mathrm{co}}^{2} \psi\right|^{2}+\left|\nabla_{\mathrm{co}}^{2} \psi\right|^{4}\right) \\
& =\frac{1}{2}\left|\nabla_{\mathrm{co}}\left(\Delta_{\mathrm{co}} \psi\right)\right|^{2}-\frac{1}{4}\left|\nabla_{\mathrm{co}}^{3} \psi\right|^{2}-64\left(C^{\prime}\right)^{2}\left(|\psi|^{2}+\left|\nabla_{\mathrm{co}} \psi\right|^{2}+\left|\nabla_{\mathrm{co}}^{2} \psi\right|^{2}+\left|\nabla_{\mathrm{co}}^{2} \psi\right|^{4}\right)
\end{aligned}
$$


This implies (3.7). By integrating the both sides of (3.7) over $\partial F$ and applying (2.6) with the associated constant $C_{\partial F}$ we obtain

$$
\begin{aligned}
\left\|\nabla_{\tau}\left(H_{F_{\psi}} \circ \Phi_{\psi}\right)\right\|_{L^{2}(\partial F)}^{2} & \geq \frac{1}{2}\left\|\nabla_{\mathrm{co}}\left(\Delta_{\mathrm{co}} \psi\right)\right\|_{L^{2}(\partial F)}^{2}-\frac{1}{4}\left\|\nabla_{\mathrm{co}}^{3} \psi\right\|_{L^{2}(\partial F)}^{2}-K^{\prime}\left(\|\psi\|_{H^{2}(\partial F)}^{2}+\left\|\nabla_{\mathrm{co}}^{2} \psi\right\|_{L^{4}(\partial F)}^{4}\right) \\
& \geq \frac{1}{4}\left\|\nabla_{\mathrm{co}}^{3} \psi\right\|_{L^{2}(\partial F)}^{2}-\left(C_{\partial F}+K^{\prime}\right)\left(\|\psi\|_{H^{2}(\partial F)}^{2}+\left\|\nabla_{\mathrm{co}}^{2} \psi\right\|_{L^{4}(\partial F)}^{4}\right) \\
& \geq \frac{1}{16}\|\psi\|_{H^{3}(\partial F)}^{2}+\frac{3}{16}\left\|\nabla_{\mathrm{co}}^{3} \psi\right\|_{L^{2}(\partial F)}^{2} \\
& -\left(C_{\partial F}+K^{\prime}+1\right)\left(\|\psi\|_{H^{2}(\partial F)}^{2}+\left\|\nabla_{\mathrm{co}}^{2} \psi\right\|_{L^{4}(\partial F)}^{4}\right)
\end{aligned}
$$

Next we interpolate the last terms in (3.18). By using Lemma 2.2 and Lemma 2.4 (recall that $\nabla_{\mathrm{co}}\left(\nabla_{\mathrm{co}} \psi\right)=\nabla_{\mathrm{co}}^{2} \psi, \nabla_{\mathrm{co}}^{2}\left(\nabla_{\mathrm{co}} \psi\right)=\nabla_{\mathrm{co}}^{3} \psi$ and $\left.\left\|\nabla_{\mathrm{co}} \psi\right\|_{L^{\infty}(\partial F} \leq\|\psi\|_{C^{1}(\partial F)}\right)$ we find a positive $M$ depending on $F$ such that

$$
\begin{aligned}
& \left\|\nabla_{\mathrm{co}} \psi\right\|_{L^{2}(\partial F)}^{2} \leq M\left\|\nabla_{\mathrm{co}}^{3} \psi\right\|_{L^{2}(\partial F)}^{\frac{2}{3}}\|\psi\|_{L^{2}(\partial F)}^{\frac{4}{3}}, \\
& \left\|\nabla_{\mathrm{co}}^{2} \psi\right\|_{L^{2}(\partial F)}^{2} \leq M\left\|\nabla_{\mathrm{co}}^{3} \psi\right\|_{L^{2}(\partial F)}^{\frac{4}{3}}\|\psi\|_{L^{2}(\partial F)}^{\frac{2}{3}} \quad \text { and } \\
& \left\|\nabla_{\mathrm{co}}^{2} \psi\right\|_{L^{4}(\partial F)}^{4} \leq M\left\|\nabla_{\mathrm{co}}^{3} \psi\right\|_{L^{2}(\partial F)}^{2}\|\psi\|_{C^{1}(\partial F)}^{2} .
\end{aligned}
$$

By applying Young's inequality on (3.19) and (3.20) we find $K^{\prime \prime}=K^{\prime \prime}\left(K^{\prime}, M, C_{\partial F}\right)$ such that

$$
\left(C_{\partial F}+K^{\prime}+1\right)\|\psi\|_{H^{2}(\partial F)}^{2} \leq \frac{1}{8}\left\|\nabla_{\mathrm{co}}^{3} \psi\right\|_{L^{2}(\partial F)}^{2}+K^{\prime \prime}\|\psi\|_{L^{2}(\partial F)}^{2} .
$$

Finally by choosing $0<\delta \leq \delta^{\prime}$ with $\left(C_{\partial F}+K^{\prime}+1\right) M \delta^{2} \leq \frac{1}{16}$ it follows from (3.18), (3.21) and (3.22), that for every $\psi \in C^{\infty}(\partial F)$ with $\|\psi\|_{C^{1}(\partial F)} \leq \delta$

$$
\left\|\nabla_{\tau}\left(H_{F_{\psi}} \circ \Phi_{\psi}\right)\right\|_{L^{2}(\partial F)}^{2} \geq \frac{1}{16}\|\psi\|_{H^{3}(\partial F)}^{2}+K^{\prime \prime}\|\psi\|_{L^{2}(\partial F)}^{2},
$$

which implies (3.6).

The previous lemma and Lemma 2.8 together yield, that for every smooth critical set $F \subset \mathbb{T}^{n}$ there are $\delta=\delta(F)$ and $K=K(F)$ such that for every $\psi \in C^{\infty}(\partial F)$ with $\|\psi\|_{C^{1}(\partial F)} \leq \delta$

$$
\begin{aligned}
\left\|\bar{H}_{F_{\psi}}-H_{F_{\psi}}\right\|_{H^{1}\left(\partial F_{\psi}\right)} & \leq K\|\psi\|_{H^{3}(\partial F)} \\
\left\|H_{F_{\psi}}\right\|_{H^{1}\left(\partial F_{\psi}\right)} & \leq K\left(\|\psi\|_{H^{3}(\partial F)}+\left|H_{F}\right|\right) .
\end{aligned}
$$

We will also use the following identities for the time derivatives of the $L^{2}$-norms of $\bar{H}_{t}-H_{t}$ and $\nabla_{\tau} H_{t}$. For the proof see Appendix.

\section{Lemma 3.4.}

Let $\left(E_{t}\right)_{t}$ be a volume preserving mean curvature flow in $\mathbb{T}^{n}$. Then for every $0 \leq t<T^{*}$

$$
\begin{aligned}
\frac{\mathrm{d}}{\mathrm{d} t}\left\|\bar{H}_{t}-H_{t}\right\|_{L^{2}\left(\partial E_{t}\right)}^{2}= & -2 \partial^{2} P\left(E_{t}\right)\left[\bar{H}_{t}-H_{t}\right]+\int_{\partial E_{t}} H_{t}\left(\bar{H}_{t}-H_{t}\right)^{3} \mathrm{~d} \mathcal{H}^{n-1} \text { and } \\
\frac{\mathrm{d}}{\mathrm{d} t}\left\|\nabla_{\tau} H_{t}\right\|_{L^{2}\left(\partial E_{t}\right)}^{2}= & -2 \int_{\partial E_{t}}\left(\Delta_{\tau} H_{t}\right)^{2}-\left(\bar{H}_{t}-H_{t}\right)\left|B_{t}\right|^{2} \Delta_{\tau} H_{t} \mathrm{~d} \mathcal{H}^{n-1} \\
& -2 \int_{\partial E_{t}}\left(\bar{H}_{t}-H_{t}\right)\left\langle\nabla_{\tau} H_{t}, B_{t} \nabla_{\tau} H_{t}\right\rangle \mathrm{d} \mathcal{H}^{n-1} \\
& +\int_{\partial E_{t}}\left|\nabla_{\tau} H_{t}\right|^{2}\left(\bar{H}_{t}-H_{t}\right) H_{t} \mathrm{~d} \mathcal{H}^{n-1} .
\end{aligned}
$$

We are now ready to prove Theorem 3.1. We divide it into four steps.

Proof of Theorem 3.1.

Step 1. We want first to utilize several lemmas and estimates we have gathered by controlling $C^{1}$ and $W^{2, p}$-norms for $1 \leq p \leq 6$. Indeed, by Remark 2.3 there is a constant $K_{0} \in \mathbb{R}_{+}$depending on $F$, such that for every $\psi \in C^{\infty}(\partial F)$ and $1 \leq p \leq 6$

$$
\|\psi\|_{C^{1}(\partial F)},\|\psi\|_{W^{2, p}(\partial F)} \leq K_{0}\|\psi\|_{H^{3}(\partial F)} .
$$

Notice that the assumption $n \leq 4$ is really needed for this conclusion. Using the estimate (3.27) we find $0<\delta<1$ and $1<K<\infty$ so that for any $\psi \in C^{\infty}(\partial F)$ with $\|\psi\|_{H^{3}(\partial F)} \leq \delta$ the norm $\|\psi\|_{C^{1}(\partial F)}$ is so small that the following four conditions hold. 
(i) Lemma 2.7 is satisfied, i.e., $F_{\psi}$ is a well-defined smooth set. Moreover, we may assume

$$
\left\|F_{\psi}, F\right\|_{C^{1}} \leq \frac{\delta_{F}}{2}
$$

where $\delta_{F}$ is as in the lemma.

(ii) The inequalities (2.10) (for $\bar{d}_{F}$, then $\bar{d}_{F} \circ \Phi_{\psi}=\psi$ ) and (3.4) are satisfied with $K$, i.e.,

$$
K^{-1}\|\psi\|_{L^{2}(\partial F)} \leq\left\|\bar{d}_{F}\right\|_{L^{2}\left(\partial F_{\psi}\right)}, \sqrt{D_{F}\left(F_{\psi}\right)} \leq K\|\psi\|_{L^{2}(\partial F)} .
$$

(iii) Lemma 3.3 and (3.23) are satisfied with $K$, i.e.,

$$
\begin{aligned}
\|\psi\|_{H^{3}(\partial F)} & \leq K\left(\left\|\nabla_{\tau} H_{F_{\psi}}\right\|_{L^{2}\left(\partial F_{\psi}\right)}+\sqrt{D_{F}\left(F_{\psi}\right)}\right) \\
\left\|\bar{H}_{F_{\psi}}-H_{F_{\psi}}\right\|_{H^{1}\left(\partial F_{\psi}\right)} & \leq K\|\psi\|_{H^{3}(\partial F)} .
\end{aligned}
$$

(iv) Lemma 2.9 is satisfied for $\bar{H}_{F_{\psi}}-H_{F_{\psi}}$ and $1 \leq p \leq 6$ with $K$, i.e.,

$$
\left\|\bar{H}_{F_{\psi}}-H_{F_{\psi}}\right\|_{L^{p}\left(\partial F_{\psi}\right)} \leq K\left\|\bar{H}_{F_{\psi}}-H_{F_{\psi}}\right\|_{H^{1}\left(\partial F_{\psi}\right)} .
$$

Moreover, we may assume $\delta$ to be so small and $K$ to be so large that by (3.27), (2.9), Lemma 2.10 and Lemma 2.14 (note that $\bar{H}_{F_{\psi}}-H_{F_{\psi}} \in T^{\perp, L^{2}}\left(\partial F_{\psi}\right)$ )

$$
\begin{aligned}
\left\|\nabla_{\tau} H_{F_{\psi}}\right\|_{L^{4}\left(\partial F_{\psi}\right)} & \leq K\left(\left\|\Delta_{\tau} H_{F_{\psi}}\right\|_{L^{2}\left(\partial F_{\psi}\right)}+\left\|\bar{H}_{F_{\psi}}-H_{F_{\psi}}\right\|_{H^{1}\left(\partial F_{\psi}\right)}\right), \\
\left\|H_{F_{\psi}}\right\|_{L^{p}\left(\partial F_{\psi}\right)},\left\|B_{F_{\psi}}\right\|_{L^{p}\left(\partial F_{\psi}\right)} & \leq K \text { for } 1 \leq p \leq 6 \text { and } \\
\sigma_{1}\left\|\bar{H}_{F_{\psi}}-H_{F_{\psi}}\right\|_{H^{1}\left(\partial F_{\psi}\right)}^{2} & \leq \partial^{2} P\left(F_{\psi}\right)\left[\bar{H}_{F_{\psi}}-H_{F_{\psi}}\right] .
\end{aligned}
$$

Next we fix the constants $C_{0}$ and $\varepsilon_{0}$ by setting

$$
\begin{aligned}
C_{0} & =\frac{4 K^{6}+1}{\sigma_{1}} \\
\varepsilon_{0} & =\min \left\{\delta, \frac{\min \left\{\sigma_{1}, 1\right\}}{16 K^{6}}\right\} .
\end{aligned}
$$

Again, for given $0<\varepsilon \leq \varepsilon_{0}$ set $\gamma_{\varepsilon}$ to be the maximal $0<s \leq \frac{\varepsilon}{2}$ satisfying

$$
\frac{2}{\sigma_{1}} \sqrt{s}+K^{2} s^{2} \leq \frac{\varepsilon^{2}}{16 K^{2}} \quad \text { and } \quad K^{2}\left(1+C_{0}\right) s \leq \frac{\varepsilon}{4}
$$

Step 2. Suppose that $\left(E_{t}\right)_{t}$ is the volume preserving mean curvature flow with a smooth initial datum $E_{0}=F_{\psi_{0}}$, where $\psi_{0} \in C^{\infty}(\partial F)$ and $\left\|\psi_{0}\right\|_{H^{3}(\partial F)} \leq \gamma_{\varepsilon}$. Since $t \mapsto\left\|E_{t}, F\right\|_{C^{1}}$ is continuous on $\left[0, T^{*}\right.$ [ (recall $T^{*}$ is the maximal lifetime), then it follows from Lemma 2.7 and (3.28) that we may write $E_{t}=F_{\psi_{t}}$ for unique $\psi_{t} \in C^{\infty}(\partial F)$ with $t \mapsto \psi_{t}$ smoothly parametrized over a short time period. Hence

$$
T_{\varepsilon}=\sup \left\{s \in \left[0, T^{*}\left[: E_{t}=F_{\psi_{t}} \text { for } \psi_{t} \in C^{\infty}(\partial F),\left\|\psi_{t}\right\|_{H^{3}(\partial F)} \leq \varepsilon \forall t \in[0, s]\right\}\right.\right.
$$

must be a positive number. The key idea is to show that the claim of theorem is satisfied for $\varepsilon$ on the time interval $\left[0, T_{\varepsilon}\right.$ [ and by virtue of the choice of $\gamma_{\varepsilon}$ we have in fact

$$
\left\|\psi_{t}\right\|_{H^{3}(\partial F)} \leq \frac{\varepsilon}{2} \quad \text { on } \quad\left[0, T_{\varepsilon}[.\right.
$$

By using a similar continuity argument as before one shows that the condition (3.39) implies $T_{\varepsilon}=T^{*}$. 
Step 3. For every $t \in\left[0, T_{\varepsilon}[\right.$

$$
\begin{aligned}
& \frac{\mathrm{d}}{\mathrm{d} t}\left\|\bar{H}_{t}-H_{t}\right\|_{L^{2}\left(\partial E_{t}\right)}^{2} \\
& \stackrel{(3.25)}{=}-2 \partial^{2} P\left(E_{t}\right)\left[\bar{H}_{t}-H_{t}\right]+\int_{\partial E_{t}} H_{t}\left(\bar{H}_{t}-H_{t}\right)^{3} \mathrm{~d} \mathcal{H}^{n-1} \\
& \stackrel{(3.35)}{\leq}-2 \sigma_{1}\left\|\bar{H}_{t}-H_{t}\right\|_{H^{1}\left(\partial E_{t}\right)}^{2}+\int_{\partial E_{t}} H_{t}\left(\bar{H}_{t}-H_{t}\right)^{3} \mathrm{~d} \mathcal{H}^{n-1} \\
& \leq-2 \sigma_{1}\left\|\bar{H}_{t}-H_{t}\right\|_{H^{1}\left(\partial E_{t}\right)}^{2}+\left\|H_{t}\right\|_{L^{4}\left(\partial E_{t}\right)}\left\|\bar{H}_{t}-H_{t}\right\|_{L^{4}\left(\partial E_{t}\right)}^{3} \\
& \stackrel{(3.34)}{\leq}-2 \sigma_{1}\left\|\bar{H}_{t}-H_{t}\right\|_{H^{1}\left(\partial E_{t}\right)}^{2}+K\left\|\bar{H}_{t}-H_{t}\right\|_{L^{4}\left(\partial E_{t}\right)}^{3} \\
& \stackrel{(3.32)}{\leq}-2 \sigma_{1}\left\|\bar{H}_{t}-H_{t}\right\|_{H^{1}\left(\partial E_{t}\right)}^{2}+K^{4}\left\|\bar{H}_{t}-H_{t}\right\|_{H^{1}\left(\partial E_{t}\right)}^{3} \\
& \stackrel{(3.31)}{\leq}\left(-2 \sigma_{1}+K^{5}\left\|\psi_{t}\right\|_{H^{3}(\partial F)}\right)\left\|\bar{H}_{t}-H_{t}\right\|_{H^{1}\left(\partial E_{t}\right)}^{2} \\
& \leq\left(-2 \sigma_{1}+K^{5} \varepsilon\right)\left\|\bar{H}_{t}-H_{t}\right\|_{H^{1}\left(\partial E_{t}\right)}^{2} \\
& \stackrel{(3.37)}{\leq}-\sigma_{1}\left\|\bar{H}_{t}-H_{t}\right\|_{H^{1}\left(\partial E_{t}\right)}^{2} \text {. }
\end{aligned}
$$

Since $-\sigma_{1}\left\|\bar{H}_{t}-H_{t}\right\|_{H^{1}\left(\partial E_{t}\right)}^{2} \leq-\sigma_{1}\left\|\bar{H}_{t}-H_{t}\right\|_{L^{2}\left(\partial E_{t}\right)}^{2}$, by using Grönwall's lemma we obtain

$$
\begin{aligned}
\left\|\bar{H}_{t}-H_{t}\right\|_{L^{2}\left(\partial E_{t}\right)}^{2} & \leq\left\|\bar{H}_{0}-H_{0}\right\|_{L^{2}\left(\partial E_{t}\right)}^{2} e^{-\sigma_{1} t} \\
& \stackrel{(3.31)}{\leq} K^{2}\left\|\psi_{0}\right\|_{H^{3}(\partial F)}^{2} e^{-\sigma_{1} t} \\
& \stackrel{(3.37)}{\leq}\left\|\psi_{0}\right\|_{H^{3}(\partial F)} e^{-\sigma_{1} t}
\end{aligned}
$$

so (3.1) holds on $\left[0, T_{\varepsilon}\left[\right.\right.$. Next we estimate $D_{F}\left(E_{t}\right)$ on $\left[0, T_{\varepsilon}[\right.$. For the time derivative

$$
\begin{aligned}
\frac{\mathrm{d}}{\mathrm{d} t} D_{F}\left(E_{t}\right) & \stackrel{(3.5)}{=} \int_{\partial E_{t}} \bar{d}_{F}\left(\bar{H}_{t}-H_{t}\right) \mathrm{d} \mathcal{H}^{n-1} \\
& \leq\left\|\bar{d}_{F}\right\|_{L^{2}\left(\partial E_{t}\right)}\left\|\bar{H}_{t}-H_{t}\right\|_{L^{2}\left(\partial E_{t}\right)} \\
& \stackrel{(3.29)}{\leq} K\left\|\psi_{t}\right\|_{L^{2}(\partial F)}\left\|\bar{H}_{t}-H_{t}\right\|_{L^{2}\left(\partial E_{t}\right)} \\
& \leq K \varepsilon\left\|\bar{H}_{t}-H_{t}\right\|_{L^{2}\left(\partial E_{t}\right)} \\
& (3.41) \\
& \leq K \varepsilon\left\|\psi_{0}\right\|_{H^{3}(\partial F)}^{\frac{1}{2}} e^{-\frac{1}{2} \sigma_{1} t} \\
& (3.37) \\
& \leq\left\|\psi_{0}\right\|_{H^{3}(\partial F)}^{\frac{1}{2}} e^{-\frac{1}{2} \sigma_{1} t} .
\end{aligned}
$$

Thus integrating over time yields

$$
\begin{aligned}
D_{F}\left(E_{t}\right) & \leq \frac{2}{\sigma_{1}}\left\|\psi_{0}\right\|_{H^{3}(\partial F)}^{\frac{1}{2}}\left(1-e^{-\frac{\sigma_{1}}{2}}\right)+D_{F}\left(E_{0}\right) \\
& \stackrel{(3.29)}{\leq} \frac{2}{\sigma_{1}}\left\|\psi_{0}\right\|_{H^{3}(\partial F)}^{\frac{1}{2}}+K^{2}\left\|\psi_{0}\right\|_{L^{2}(\partial F)}^{2} \\
& \stackrel{(3.38)}{\leq} \frac{\varepsilon^{2}}{16 K^{2}}
\end{aligned}
$$

Step 4. In this last step we finish the proof by showing that (3.2) and (3.39) are satisfied on $\left[0, T_{\varepsilon}[\right.$. To this end we have to estimate $\frac{\mathrm{d}}{\mathrm{d} t}\left\|\nabla_{\tau} H_{t}\right\|_{L^{2}\left(\partial E_{t}\right)}^{2}$ on $\left[0, T_{\varepsilon}[\right.$. Recall that by (3.26) we have

$$
\begin{aligned}
\frac{\mathrm{d}}{\mathrm{d} t}\left\|\nabla_{\tau} H_{t}\right\|_{L^{2}\left(\partial E_{t}\right)}^{2} & =-2 \int_{\partial E_{t}}\left(\Delta_{\tau} H_{t}\right)^{2}-\left(\bar{H}_{t}-H_{t}\right)\left|B_{t}\right|^{2} \Delta_{\tau} H_{t} \mathrm{~d} \mathcal{H}^{n-1} \\
& -2 \int_{\partial E_{t}}\left(\bar{H}_{t}-H_{t}\right)\left\langle\nabla_{\tau} H_{t}, B_{t} \nabla_{\tau} H_{t}\right\rangle \mathrm{d} \mathcal{H}^{n-1}+\int_{\partial E_{t}}\left|\nabla_{\tau} H_{t}\right|^{2}\left(\bar{H}_{t}-H_{t}\right) H_{t} \mathrm{~d} \mathcal{H}^{n-1} \\
& =T_{1}+T_{2}+T_{3}
\end{aligned}
$$


Next we estimate the terms $T_{1}, T_{2}$ and $T_{3}$. First we have

Second

$$
\begin{aligned}
T_{1} & \stackrel{\text { Young }}{\leq}-\left\|\Delta_{\tau} H_{t}\right\|_{L^{2}\left(\partial E_{t}\right)}^{2}+4 \int_{\partial E_{t}}\left|\bar{H}_{t}-H_{t}\right|^{2}\left|B_{t}\right|^{4} \mathrm{~d} \mathcal{H}^{n-1} \\
& \leq-\left\|\Delta_{\tau} H_{t}\right\|_{L^{2}\left(\partial E_{t}\right)}^{2}+4\left\|\bar{H}_{t}-H_{t}\right\|_{L^{6}\left(\partial E_{t}\right)}^{2}\left\|B_{t}\right\|_{L^{6}\left(\partial E_{t}\right)}^{4} \\
& \quad(3.34) \\
& \leq\left\|\Delta_{\tau} H_{t}\right\|_{L^{2}\left(\partial E_{t}\right)}^{2}+4 K^{4}\left\|\bar{H}_{t}-H_{t}\right\|_{L^{6}\left(\partial E_{t}\right)}^{2} \\
& \quad(3.32) \\
\quad & -\left\|\Delta_{\tau} H_{t}\right\|_{L^{2}\left(\partial E_{t}\right)}^{2}+4 K^{6}\left\|\bar{H}_{t}-H_{t}\right\|_{H^{1}\left(\partial E_{t}\right)}^{2} .
\end{aligned}
$$

$$
\begin{aligned}
& T_{2} \leq 2 \int_{\partial E_{t}}\left|\bar{H}_{t}-H_{t}\right|\left|\nabla_{\tau} H_{t}\right|^{2}\left|B_{t}\right| \mathrm{d} \mathcal{H}^{n-1} \\
& \leq 2\left\|\nabla_{\tau} H_{t}\right\|_{L^{4}\left(\partial E_{t}\right)}^{2}\left\|\bar{H}_{t}-H_{t}\right\|_{L^{4}\left(\partial E_{t}\right)}\left\|B_{t}\right\|_{L^{4}\left(\partial E_{t}\right)} \\
& \text { (3.34) } \\
& \stackrel{(3.34)}{\leq} 2 K\left\|\nabla_{\tau} H_{t}\right\|_{L^{4}\left(\partial E_{t}\right)}^{2}\left\|\bar{H}_{t}-H_{t}\right\|_{L^{4}\left(\partial E_{t}\right)} \\
& \stackrel{(3.33)}{\leq} 2 K^{3}\left(\left\|\Delta_{\tau} H_{t}\right\|_{L^{2}\left(\partial E_{t}\right)}+\left\|\bar{H}_{t}-H_{t}\right\|_{H^{1}\left(\partial E_{t}\right)}\right)^{2}\left\|\bar{H}_{t}-H_{t}\right\|_{L^{4}\left(\partial E_{t}\right)} \\
& \stackrel{(3.32)}{\leq} 2 K^{4}\left\|\bar{H}_{t}-H_{t}\right\|_{H^{1}\left(\partial E_{t}\right)}\left(\left\|\Delta_{\tau} H_{t}\right\|_{L^{2}\left(\partial E_{t}\right)}+\left\|\bar{H}_{t}-H_{t}\right\|_{H^{1}\left(\partial E_{t}\right)}\right)^{2} \\
& \stackrel{(3.31)}{\leq} 2 K^{5}\left\|\psi_{t}\right\|_{H^{3}(\partial F)}\left(\left\|\Delta_{\tau} H_{t}\right\|_{L^{2}\left(\partial E_{t}\right)}+\left\|\bar{H}_{t}-H_{t}\right\|_{H^{1}\left(\partial E_{t}\right)}\right)^{2} \\
& \leq 4 K^{5}\left\|\psi_{t}\right\|_{H^{3}(\partial F)}\left\|\Delta_{\tau} H_{t}\right\|_{L^{2}\left(\partial E_{t}\right)}^{2}+4 K^{5}\left\|\psi_{t}\right\|_{H^{3}(\partial F)}\left\|\bar{H}_{t}-H_{t}\right\|_{H^{1}\left(\partial E_{t}\right)}^{2} \\
& \leq 4 K^{5} \varepsilon\left\|\Delta_{\tau} H_{t}\right\|_{L^{2}\left(\partial E_{t}\right)}^{2}+4 K^{5} \varepsilon\left\|\bar{H}_{t}-H_{t}\right\|_{H^{1}\left(\partial E_{t}\right)}^{2} \\
& \stackrel{(3.37)}{\leq} \frac{1}{4}\left\|\Delta_{\tau} H_{t}\right\|_{L^{2}\left(\partial E_{t}\right)}^{2}+\frac{1}{2}\left\|\bar{H}_{t}-H_{t}\right\|_{H^{1}\left(\partial E_{t}\right)}^{2} \text {. }
\end{aligned}
$$

Finally by estimating in a similar way as above we obtain

$$
T_{3} \leq \frac{1}{8}\left\|\Delta_{\tau} H_{t}\right\|_{L^{2}\left(\partial E_{t}\right)}^{2}+\frac{1}{4}\left\|\bar{H}_{t}-H_{t}\right\|_{H^{1}\left(\partial E_{t}\right)}^{2} .
$$

Hence (3.43), (3.44) and (3.45) together yield

$$
\begin{aligned}
\frac{\mathrm{d}}{\mathrm{d} t}\left\|\nabla_{\tau} H_{t}\right\|_{L^{2}\left(\partial E_{t}\right)}^{2} & \leq-\frac{1}{2}\left\|\Delta_{\tau} H_{t}\right\|_{L^{2}\left(\partial E_{t}\right)}^{2}+\left(4 K^{6}+1\right)\left\|\bar{H}_{t}-H_{t}\right\|_{H^{1}\left(\partial E_{t}\right)}^{2} \\
& \stackrel{(3.36)}{=}-\frac{1}{2}\left\|\Delta_{\tau} H_{t}\right\|_{L^{2}\left(\partial E_{t}\right)}^{2}+\sigma_{1} C_{0}\left\|\bar{H}_{t}-H_{t}\right\|_{H^{1}\left(\partial E_{t}\right)}^{2}
\end{aligned}
$$

on $\left[0, T_{\varepsilon}\left[\right.\right.$. Then the previous estimate with (3.40) yields that for every $t \in\left[0, T_{\varepsilon}[\right.$

$$
\frac{\mathrm{d}}{\mathrm{d} t}\left[\left\|\nabla_{\tau} H_{t}\right\|_{L^{2}\left(\partial E_{t}\right)}^{2}+C_{0}\left\|\bar{H}_{t}-H_{t}\right\|_{L^{2}\left(\partial E_{t}\right)}^{2}\right] \leq-\frac{1}{2}\left\|\Delta_{\tau} H_{t}\right\|_{L^{2}\left(\partial E_{t}\right)}^{2},
$$

which implies (3.2). In particular

$$
t \mapsto\left\|\nabla_{\tau} H_{t}\right\|_{L^{2}\left(\partial E_{t}\right)}^{2}+C_{0}\left\|\bar{H}_{t}-H_{t}\right\|_{L^{2}\left(\partial E_{t}\right)}^{2}
$$

is decreasing map on $\left[0, T_{\varepsilon}[\right.$ and therefore

$$
\left\|\nabla_{\tau} H_{t}\right\|_{L^{2}\left(\partial E_{t}\right)} \leq\left\|\nabla_{\tau} H_{0}\right\|_{L^{2}\left(\partial E_{0}\right)}+C_{0}\left\|\bar{H}_{0}-H_{0}\right\|_{L^{2}\left(\partial E_{0}\right)} .
$$

Finally for every $t \in\left[0, T_{\varepsilon}[\right.$

$$
\begin{aligned}
& \left\|\psi_{t}\right\|_{H^{3}\left(\partial E_{t}\right)} \stackrel{(3.30)}{\leq} K\left(\left\|\nabla_{\tau} H_{t}\right\|_{L^{2}\left(\partial E_{t}\right)}+\sqrt{D_{F}\left(E_{t}\right)}\right) \\
& \stackrel{(3.46)}{\leq} K\left(\left\|\nabla_{\tau} H_{0}\right\|_{L^{2}\left(\partial E_{0}\right)}+C_{0}\left\|\bar{H}_{0}-H_{0}\right\|_{L^{2}\left(\partial E_{0}\right)}\right)+K \sqrt{D_{F}\left(E_{t}\right)} \\
& \stackrel{(3.42)}{\leq} K\left(1+C_{0}\right)\left\|\bar{H}_{0}-H_{0}\right\|_{H^{1}\left(\partial E_{0}\right)}+\frac{\varepsilon}{4} \\
& \stackrel{(3.31)}{\leq} K^{2}\left(1+C_{0}\right)\left\|\psi_{0}\right\|_{H^{3}\left(\partial E_{t}\right)}+\frac{\varepsilon}{4} \\
& \stackrel{(3.38)}{\leq} \frac{\varepsilon}{2}
\end{aligned}
$$




\section{The main Result}

In this section we will prove the main result. We give first the technical statement of the theorem in contrast to the heuristical one we presented in Introduction.

\section{Theorem 4.1 (Main Theorem).}

Let $\mathbb{T}^{n}$ be a flat torus with $n=3,4$ and assume that $F \subset \mathbb{T}^{n}$ is a strictly stable set. There exist positive constants $\delta_{0}, \sigma_{0} \in \mathbb{R}_{+}$depending on $F$ such that the following hold.

If $E_{0}$ is a smooth set in $\mathbb{T}^{n}$ with $\left|E_{0}\right|=|F|$ of the form $E_{0}=F_{\psi_{0}}$, where $\psi_{0} \in C^{\infty}(\partial F)$ and $\left\|\psi_{0}\right\|_{H^{3}(\partial F)} \leq \delta_{0}$, then the volume preserving mean curvature flow $\left(E_{t}\right)_{t}$ in $\mathbb{T}^{n}$ with the initial datum $E_{0}$ satisfies the following conditions.

(i) The flow has infinite lifetime.

(ii) There exist $p=p\left(F, E_{0}\right) \in \mathbb{R}^{n}$ and $C=C(F) \in \mathbb{R}_{+}$such that the flow converges to $F+p$ exponentially fast in $W^{2,5}$-sense, that is, $E_{t}=(F+p)_{\varphi_{t}}$ for $\varphi_{t} \in C^{\infty}(\partial(F+p))$ and $\left\|\varphi_{t}\right\|_{W^{2,5}} \leq C e^{-\sigma_{0} t}$.

(iii) $|p| \rightarrow 0$ and $C \rightarrow 0$ as $\left\|\psi_{0}\right\|_{H^{3}(\partial F)} \rightarrow 0$.

\section{Remark 4.2.}

In the statement of the main theorem the $W^{2,5}$-convergence can be replaced by $W^{2, q}$-convergence, where $1 \leq q<\infty$, if $n=3$, and $1 \leq q<6$, if $n=4$. In this case the proof would be similar to the original proof.

The main idea of the proof is obviously to employ the short time existence (Theorem 2.15) and the monotonicity result (Theorem 3.1).

\section{Proof of the Main Theorem.}

Let $F \subset \mathbb{T}^{n}$ be a strictly stable set and let us fix the constants $\delta_{0}$ and $\sigma_{0}$ for $F$ as in the statement of the main theorem. Let $\varepsilon_{0}, \sigma_{1} \in \mathbb{R}_{+}$and $0<\gamma_{\varepsilon}<\varepsilon$ (for every $0<\varepsilon \leq \varepsilon_{0}$ ) for $F$ be as in Theorem 3.1. Choose first a positive $c$ so small that the following hold.

(i) The condition $\|\psi\|_{C^{1}(\partial F)} \leq c$ for $\psi \in C^{1, \frac{1}{4}}(\partial F)$ implies via Lemma 2.7 that the set $E_{\psi}$ is defined as a $C^{1, \frac{1}{4}}$-set, the map $\Phi_{\psi}$ defined as in the same lemma is a $C^{1, \frac{1}{4}}$-diffeomorphism from $\partial F$ to $\partial F_{\psi}$ and

$$
K^{-1}|\partial F| \leq\left|\partial F_{\psi}\right| \leq K|\partial F|
$$

for some real number $K \geq 1$ depending on $c$ and $F$.

(ii) Further, if $\psi$ is smooth, the same condition implies via (3.24) that by increasing $K$, if necessary,

$$
\left\|H_{F_{\psi}}\right\|_{H^{1}\left(F_{\psi}\right)} \leq K\left(\|\psi\|_{H^{3}(\partial F)}+\left|H_{F}\right|\right) .
$$

(iii) Since a translate of $F$ satisfies Lemma 3.3 and (3.4) with the same bounds as $F$, then by using the previous lemmas and Lemma 2.7, possibly decreasing $c$ and increasing $K$, we obtain the following. Assume $F+p=F_{g}$ with $g \in C^{\infty}(\partial F)$ and $\|g\|_{C^{1}(\partial F)} \leq c$. Then for every $\psi \in C^{\infty}(\partial F)$ with $\|\psi\|_{C^{1}(\partial F)} \leq c$ there is a unique $\varphi \in C^{\infty}(\partial(F+p))$ such that $F_{\psi}=(F+p)_{\varphi}$ and we have the following uniform estimates

$$
\begin{aligned}
K^{-1} \sqrt{D_{F+p}\left(F_{\psi}\right)} & \leq\|\varphi\|_{L^{2}(\partial(F+p))} \leq K \sqrt{D_{F+p}\left(F_{\psi}\right)} \text { and } \\
K^{-1}\left\|\nabla_{\tau} H_{F_{\psi}}\right\|_{L^{2}\left(\partial F_{\psi}\right)} & \leq\|\varphi\|_{H^{3}(\partial(F+p))} \leq K\left(\left\|\nabla_{\tau} H_{F_{\psi}}\right\|_{L^{2}\left(\partial F_{\psi}\right)}+\sqrt{D_{F+p}\left(F_{\psi}\right)}\right) .
\end{aligned}
$$

(iv) Finally the condition $\|\psi\|_{C^{1, \frac{1}{4}(\partial F)}} \leq c$ for $\psi \in C^{\infty}(\partial F)$ means that Theorem 2.15 (in the case $\beta=\frac{1}{4}$ ) is satisfied for the initial set $E_{\psi}$.

Next choose $0<\varepsilon_{1} \leq \varepsilon_{0}$ such that the condition $\|\psi\|_{H^{3}(\partial F)} \leq \varepsilon_{1}$ for $\psi \in C^{\infty}(\partial F)$ means that first Lemma 2.13 is satisfied, provided that $F_{\psi}$ is critical with $\left|F_{\psi}\right|=|F|$, and second via Lemma 2.6 $\|\psi\|_{C^{1}(\partial F)} \leq\|\psi\|_{C^{1, \frac{1}{4}(\partial F)}} \leq c$ (here we really need the assumption $\left.n \leq 4\right)$. At this point we set

$$
\delta_{0}=\gamma_{\varepsilon_{1}} \quad \text { and } \quad \sigma_{0}=-\frac{\sigma_{1}}{2}\left(\frac{1}{3}-\frac{n-1}{10}\right) .
$$

Fix an arbitrary $\psi_{0} \in C^{\infty}(\partial F)$ with $\left\|\psi_{0}\right\|_{H^{3}(\partial F)} \leq \delta_{0}$ and $\left|F_{\psi_{0}}\right|=|F|$. Then by Theorem 2.15 there exists a unique volume preserving mean curvature flow $\left(E_{t}\right)_{t}$ starting from $E_{0}=F_{\psi_{0}}$ and the maximal lifetime $T^{*}$ is bounded from below by $T=T\left(F, \frac{1}{4}\right)>0$ as in Theorem 2.15. Again, by Theorem 3.1 we may write $E_{t}=F_{\psi_{t}}$, where $\psi_{t} \in C^{\infty}(\partial F)$ with $\left\|\psi_{t}\right\|_{H^{3}(\partial F)} \leq \varepsilon_{1}$, and the 
inequalities (3.1) and (3.2) are satisfied for every $t \in\left[0, T^{*}[\right.$. We divide the proof into three steps, whose statements are listed below.

Step 1. The flow $\left(E_{t}\right)_{t}$ has infinite lifetime and there exists $\psi_{\infty} \in H^{3}(\partial F)$ with $\left\|\psi_{\infty}\right\|_{H^{3}(\partial F)} \leq \varepsilon$ such that $\psi_{t} \rightarrow \psi_{\infty}$ in $C^{1, \frac{1}{4}}(\partial F)$. Further, there exist a positive constant $C_{F}$ independent of the choice of $\psi_{0}$ and an increasing $\rho:\left[0, \delta_{0}\right] \rightarrow\left[0, \infty\left[\right.\right.$ with $\lim _{s \rightarrow 0+} \rho(s)=0$ such that every $t \in[0, \infty[$

$$
\begin{aligned}
D_{E_{\infty}}\left(E_{t}\right) & \leq C_{F}\left\|\psi_{0}\right\|_{H^{3}(\partial F)} e^{-\sigma_{1} t} \text { and } \\
\left\|\psi_{\infty}\right\|_{L^{\infty}(\partial F)} & \leq \rho\left(\left\|\psi_{0}\right\|_{H^{3}(\partial F)}\right),
\end{aligned}
$$

where $E_{\infty}=F_{\psi_{\infty}}$ is the corresponding $C^{1, \frac{1}{4}}$-limit set.

Step 2. The limit set is of the form $E_{\infty}=F+p$, where $p \rightarrow 0$ as $\left\|\psi_{0}\right\|_{H^{3}(\partial F)} \rightarrow 0$.

Step 3. The $W^{2,5}$-convergence of the flow: For each $t \in\left[0, \infty\left[\right.\right.$ there is $\varphi_{t} \in C^{\infty}\left(\partial E_{\infty}\right)$ with $E_{t}=\left(E_{\infty}\right)_{\varphi_{t}}$ and $\left\|\varphi_{t}\right\|_{W^{2,5}(\partial F)} \leq C e^{-\sigma_{0} t}$, where $C$ is independent of the choice of $\psi_{0}$ and $C \rightarrow 0$ as $\left\|\psi_{0}\right\|_{H^{3}(\partial F)} \rightarrow 0$.

Since $\psi_{0} \in C^{\infty}(\partial F)$ with $\left\|\psi_{0}\right\|_{H^{3}(\partial F)} \leq \delta_{0}$ was arbitrarily chosen, the claim of theorem follows immediately from these statements. Let us prove them in order as listed.

Proof of Step 1. Assume by contradiction $T^{*}<\infty$ and choose $\hat{t} \in\left[0, T^{*}\left[\right.\right.$ such that $T^{*}-\hat{t}<T$, where $T=T\left(F, \frac{1}{4}\right)$ as in Theorem 2.15. Now $\left\|\psi_{\hat{t}}\right\|_{H^{3}(\partial F)} \leq \varepsilon_{1}$ so $\left\|\psi_{\hat{t}}\right\|_{C^{1, \frac{1}{4}(\partial F)}} \leq c$ and hence by Theorem 2.15 there exists a unique volume preserving mean curvature flow $\left(\hat{E}_{t}\right)_{t}$ starting from $E_{\hat{t}}$ with a maximal lifetime at least $T$. It follows from the uniqueness and from the semi-group property of $\left(E_{t}\right)_{t}$ that $E_{t}=\hat{E}_{t-\hat{t}}$ for every $t \in\left[\hat{t}, T^{*}\left[\right.\right.$. This means that the flow $\left(E_{t}\right)_{t}$ can be extended beyond $T^{*}$, which contradicts its maximality. Therefore it holds $T^{*}=\infty$.

Take a sequence $\left(t_{k}\right)_{k=1}^{\infty} \subset \mathbb{R}_{+}$with $t_{k} \rightarrow \infty$. Since $\left\|\psi_{t_{k}}\right\|_{H^{3}(\partial F)} \leq \varepsilon_{1}$ for every $k$ and $H^{3}(\partial F)$ is weakly compact, then, up to a subsequence, there is a weak limit $\psi_{\infty} \in H^{3}(\partial F)$ with $\left\|\psi_{\infty}\right\|_{H^{3}(\partial F)} \leq$ $\varepsilon_{1}$. Further, it follows from Lemma 2.6 that the sequence converges to $\psi_{\infty}$ in $C^{1, \frac{1}{4}}(\partial F)$. Now $C^{1}-$ convergence implies that $\left\|\psi_{\infty}\right\|_{C^{1}(\partial F)} \leq c$ so $E_{\infty}:=F_{\psi_{\infty}}$ is defined as a $C^{1, \frac{1}{4}}$-set and the map $\Phi_{\psi_{\infty}}$ is a $C^{1, \frac{1}{4}}$-diffeomorphism from $\partial F$ to $\partial E_{\infty}$.

Next we check that (4.6) holds for every $t$. Notice first that $\left|E_{t_{k}} \Delta E_{\infty}\right| \rightarrow 0$, which implies $D_{E_{\infty}}\left(E_{t_{k}}\right) \rightarrow 0$. For a fixed $t \in\left[0, \infty\left[\right.\right.$ and every $t_{k}>t$ we may estimate

$$
\begin{aligned}
\left|D_{E_{\infty}}\left(E_{t_{k}}\right)-D_{E_{\infty}}\left(E_{t}\right)\right| & =\left|\int_{t}^{t_{k}} \frac{\mathrm{d}}{\mathrm{d} s} D_{E_{\infty}}\left(E_{s}\right) \mathrm{d} s\right| \\
& \stackrel{(3.5)}{=}\left|\int_{t}^{t_{k}} \int_{\partial E_{s}} \bar{d}_{E_{\infty}}\left(\bar{H}_{s}-H_{s}\right) \mathrm{d} \mathcal{H}^{n-1} \mathrm{~d} s\right| \\
& \leq \int_{t}^{t_{k}}\left\|\bar{d}_{E_{\infty}}\right\|_{L^{2}\left(\partial E_{s}\right)}\left\|\bar{H}_{s}-H_{s}\right\|_{L^{2}\left(\partial E_{s}\right)} \mathrm{d} s \\
& \leq \sqrt{n} \int_{t}^{t_{k}}\left|\partial E_{s}\right|\left\|\bar{H}_{s}-H_{s}\right\|_{L^{2}\left(\partial E_{s}\right)} \mathrm{d} s \\
& \left(\stackrel{4.1)}{\leq} \sqrt{n} K|\partial F| \int_{t}^{t_{k}}\left\|\bar{H}_{s}-H_{s}\right\|_{L^{2}\left(\partial E_{s}\right)} \mathrm{d} s\right. \\
& \stackrel{(3.1)}{\leq} \sqrt{n} K|\partial F|\left\|\psi_{0}\right\|_{H^{3}(\partial F)} \int_{t}^{t_{k}} e^{-\sigma_{1} s} \mathrm{~d} s \\
& \leq \frac{\sqrt{n} K|\partial F|}{\sigma_{1}}\left\|\psi_{0}\right\|_{H^{3}(\partial F)} e^{-\sigma_{1} t} .
\end{aligned}
$$

Since $D_{E_{\infty}}\left(E_{t_{k}}\right) \rightarrow 0$, then the previous estimate implies (4.6) for $t$. By doing a similar estimate for $D_{F}\left(E_{t_{k}}\right)-D_{F}\left(E_{0}\right)$, using (4.3) for $F$ and Lemma (4.7) and recalling that $\left\|\psi_{t_{k}}\right\|_{H^{3}(\partial F)} \leq \varepsilon_{1}$ we find a constant $\tilde{C}_{F}$ not depending on the choice of $\psi_{0}$ such that

$$
\left\|\psi_{t_{k}}\right\|_{L^{\infty}(\partial F)} \leq \tilde{C}_{F}\left\|\psi_{0}\right\|_{H^{3}(\partial F)}^{\frac{1}{2}\left(1-\frac{n-1}{6}\right)} .
$$

Thus by passing to limit we see that (4.7) holds for $\psi_{\infty}$. Finally we check that the full $C^{1, \frac{1}{4}}$ convergence in time holds. To this end, it suffices to show that every sequence $\left(\tilde{t}_{k}\right)_{k=1}^{\infty} \subset \mathbb{R}_{+}$with $\tilde{t}_{k} \rightarrow \infty$ has a subsequence converging to $\psi_{\infty}$ in $C^{1, \frac{1}{4}}(\partial F)$. Indeed, by arguing as previously in a case of such sequence $\left(\tilde{t}_{k}\right)_{k=1}^{\infty} \subset \mathbb{R}_{+}$, we find a subsequence converging to some limit $\tilde{\psi}_{\infty} \in H^{3}(\partial F)$ 
in $C^{1, \frac{1}{4}}(\partial F)$ and $F_{\tilde{\psi}_{\infty}}$ is defined as a $C^{1, \frac{1}{4}}$-set. We may again assume that the subsequence is the whole sequence and hence $\left|E_{\tilde{t}_{k}} \Delta F_{\tilde{\psi}_{\infty}}\right| \rightarrow 0$, which implies together (4.6) and the boundedness of $\bar{d}_{E_{\infty}}$ that

$$
D_{E_{\infty}}\left(F_{\tilde{\psi}_{\infty}}\right)=\lim _{k} D_{E_{\infty}}\left(E_{\tilde{t}_{k}}\right)=0
$$

This implies that $E_{\infty}=F_{\psi_{\infty}}$ and further $\psi_{\infty}=\tilde{\psi}_{\infty}$. Thus the first step has been concluded.

Proof of Step 2. First we show that $E_{\infty}$ is a smooth and critical set. Since $E_{\infty}$ is a $C^{1, \frac{1}{4}}$-set, then thanks to Lemma 2.1 it suffices to show it to be stationary. We need to find $\lambda_{\infty} \in \mathbb{R}$ such that for every $f \in C^{\infty}\left(\mathbb{T}^{n} ; \mathbb{R}^{n}\right)$

$$
\int_{\partial E_{\infty}} \operatorname{div}_{\tau} f \mathrm{~d} \mathcal{H}^{n-1}=\lambda_{\infty} \int_{\partial E_{\infty}}\left\langle f, \nu_{\infty}\right\rangle \mathrm{d} \mathcal{H}^{n-1},
$$

where $\nu_{\infty}$ is the corresponding unit normal field of $\partial E_{\infty}$ with inside-out orientation. Since $\psi_{t} \rightarrow \psi_{\infty}$ in $C^{1}(\partial F)$, then $\Phi_{\psi_{t}} \rightarrow \Phi_{\psi_{\infty}}, \nu_{t} \circ \Phi_{\psi_{t}} \rightarrow \nu_{\infty} \circ \Phi_{\psi_{\infty}}$ and $J_{\tau} \Phi_{\psi_{t}} \rightarrow J_{\tau} \Phi_{\psi_{\infty}}$ uniformly on $\partial F$. Thus by using the change of variables formula we obtain for every $f \in C^{\infty}\left(\mathbb{T}^{n} ; \mathbb{R}^{n}\right)$

$$
\begin{gathered}
\int_{\partial E_{t}} \operatorname{div}_{\tau} f \mathrm{~d} \mathcal{H}^{n-1} \longrightarrow \int_{\partial E_{\infty}} \operatorname{div}_{\tau} f \mathrm{~d} \mathcal{H}^{n-1} \text { and } \\
\int_{\partial E_{t}}\left\langle f, \nu_{t}\right\rangle \mathrm{d} \mathcal{H}^{n-1} \longrightarrow \int_{\partial E_{\infty}}\left\langle f, \nu_{\infty}\right\rangle \mathrm{d} \mathcal{H}^{n-1} .
\end{gathered}
$$

By using (4.1), (4.2) and Hölder's inequality we see that $\bar{H}_{t}$ is bounded in time and hence we find a sequence $\left(\bar{H}_{t_{k}}\right)_{k}, t_{k} \rightarrow \infty$, converging to some real number say $\lambda_{\infty}$. By the divergence theorem

$$
\begin{aligned}
\int_{\partial E_{t_{k}}} \operatorname{div}_{\tau} f \mathrm{~d} \mathcal{H}^{n-1} & =\int_{\partial E_{t_{k}}} H_{t_{k}}\left\langle f, \nu_{t}\right\rangle \mathrm{d} \mathcal{H}^{n-1} \\
& =\bar{H}_{t_{k}} \int_{\partial E_{t_{k}}}\left\langle f, \nu_{t_{k}}\right\rangle \mathrm{d} \mathcal{H}^{n-1}+\int_{\partial E_{t_{k}}}\left(H_{t_{k}}-\bar{H}_{t_{k}}\right)\left\langle f, \nu_{t}\right\rangle \mathrm{d} \mathcal{H}^{n-1}
\end{aligned}
$$

and thus by letting $t_{k} \rightarrow \infty$ and recalling (4.9) and (4.10) we obtain (4.8), since

$$
\begin{aligned}
\left|\int_{\partial E_{t_{k}}}\left(H_{t_{k}}-\bar{H}_{t_{k}}\right)\left\langle f, \nu_{t_{k}}\right\rangle \mathrm{d} \mathcal{H}^{n-1}\right| & \leq \sup _{\mathbb{T}^{n}}\left|f\left\|\left.\partial E_{t_{k}}\right|^{\frac{1}{2}}\right\|\left(H_{t_{k}}-\bar{H}_{t_{k}}\right) \|_{L^{2}\left(\partial E_{t_{k}}\right)}\right. \\
& \stackrel{(4.1)}{\leq} \sup _{\mathbb{T}^{n}}|f|(K|\partial F|)^{\frac{1}{2}}\left\|\left(H_{t_{k}}-\bar{H}_{t_{k}}\right)\right\|_{L^{2}\left(\partial E_{t_{k}}\right)} \\
& \stackrel{(3.1)}{\leq} \sup _{\mathbb{T}^{n}}|f|\left(K|\partial F|\left\|\psi_{0}\right\|_{H^{3}(\partial F)} e^{-\sigma_{1} t_{k}}\right)^{\frac{1}{2}} .
\end{aligned}
$$

Thus $E_{\infty}$ is a smooth and critical set and since $\left\|\psi_{\infty}\right\|_{H^{3}(\partial F)} \leq \varepsilon_{1}$ (recall the choice of $\varepsilon_{1}$ ) and $\left|E_{\infty}\right|=|F|$ (by (4.4)), it follows from Lemma 2.13 that $E_{\infty}=\bar{F}+p$ for some $p \in \mathbb{R}^{n}$. Since now $d_{H}\left(F, E_{\infty}\right) \leq\left\|\psi_{\infty}\right\|_{L^{\infty}(\partial F)}$, then it follows from (4.7) that $d_{H}\left(F, E_{\infty}\right) \rightarrow 0$ as $\left\|\psi_{0}\right\|_{H^{3}(\partial F)}$ tends to zero. This implies that we may choose $p$ in such a way that simultaneously $p \rightarrow 0$.

Proof of Step 3. Since now $F+p=E_{\infty}$ and $\left\|\psi_{\infty}\right\|_{C^{1}(\partial E)},\left\|\psi_{t}\right\|_{C^{1}(\partial E)} \leq c$, then by (iii) we may write $\partial E_{t}$ as a smooth graph in normal direction over $\partial(F+p)$, i.e., for every $t \in[0, \infty[$ there is a unique $\varphi_{t} \in C^{\infty}\left(\partial E_{\infty}\right)$ for which $E_{t}=\left(E_{\infty}\right)_{\varphi_{t}}$. Again, for every $t \in[0, \infty[$

$$
\left\|\varphi_{t}\right\|_{L^{2}\left(\partial E_{\infty}\right)} \stackrel{(4.3)}{\leq} K \sqrt{D_{E_{\infty}}\left(E_{t}\right)} \stackrel{(4.6)}{\leq} K C_{F}^{\frac{1}{2}} e^{-\frac{\sigma_{1}}{2} t}
$$


and

$$
\begin{aligned}
\left\|\varphi_{t}\right\|_{H^{3}\left(\partial E_{\infty}\right)} & \stackrel{(4.4)}{\leq} K\left(\left\|\nabla_{\tau} H_{t}\right\|_{L^{2}\left(\partial E_{t}\right)}+\sqrt{D_{E_{\infty}}\left(E_{t}\right)}\right) \\
& \stackrel{(3.2)}{\leq} K\left(\left\|\nabla_{\tau} H_{0}\right\|_{L^{2}\left(\partial E_{0}\right)}+C_{0}^{\frac{1}{2}}\left\|\bar{H}_{0}-H_{0}\right\|_{L^{2}\left(\partial E_{0}\right)}+\sqrt{D_{E_{\infty}}\left(E_{t}\right)}\right) \\
& \stackrel{(4.4)}{\leq} K\left(K\left\|\psi_{0}\right\|_{H^{3}(\partial F)}+C_{0}^{\frac{1}{2}}\left\|\bar{H}_{0}-H_{0}\right\|_{L^{2}\left(\partial E_{0}\right)}+\sqrt{D_{E_{\infty}\left(E_{t}\right)}}\right) \\
& \stackrel{(3.1)}{\leq} K\left(K\left\|\psi_{0}\right\|_{H^{3}(\partial F)}+C_{0}^{\frac{1}{2}}\left\|\psi_{0}\right\|_{H^{3}(\partial F)}^{\frac{1}{2}}+\sqrt{D_{E_{\infty}}\left(E_{t}\right)}\right) \\
& \stackrel{(4.6)}{\leq} K\left(K\left\|\psi_{0}\right\|_{H^{3}(\partial F)}+C_{0}^{\frac{1}{2}}\left\|\psi_{0}\right\|_{H^{3}(\partial F)}^{\frac{1}{2}}+C_{F}^{\frac{1}{2}}\left\|\psi_{0}\right\|_{H^{3}(\partial F)}^{\frac{1}{2}}\right) \\
& \leq K\left(K \varepsilon_{1}^{\frac{1}{2}}+C_{0}^{\frac{1}{2}}+C_{F}^{\frac{1}{2}}\right)\left\|\psi_{0}\right\|_{H^{3}(\partial F)}
\end{aligned}
$$

This means that there exists a positive constant $C_{F}^{\prime}$ independent of the choice of $\psi_{0}$ such that $\left\|\varphi_{t}\right\|_{L^{2}\left(\partial E_{\infty}\right)} \leq C_{F}^{\prime}\left\|\psi_{0}\right\|_{H^{3}(\partial F)}^{\frac{1}{2}} e^{-\frac{\sigma_{1}}{2} t}$ and $\left\|\varphi_{t}\right\|_{H^{3}\left(\partial E_{\infty}\right)} \leq C_{F}^{\prime}\left\|\psi_{0}\right\|_{H^{3}(\partial F)}^{\frac{1}{2}}$ for every $t \in[0, \infty[$. Since $\partial(F+p)$ shares same interpolation bounds than $\partial F$, then by using the previous estimates and Lemma 2.2 in the case

$$
\frac{1}{5}=\frac{j}{n-1}+\left(\frac{1}{2}-\frac{3}{n-1}\right) \alpha+\frac{1-\alpha}{2}
$$

with $\alpha=\frac{j}{3}+\frac{n-1}{10}, j=0,1,2$ and a corresponding interpolation constant (which we may assume to be the same $C_{F}^{\prime}$ ) we obtain

$$
\begin{aligned}
\left\|\nabla_{\mathrm{co}}^{j} \varphi_{t}\right\|_{L^{5}\left(\partial E_{\infty}\right)} & \leq C_{F}^{\prime}\left\|\nabla_{\mathrm{co}}^{3} \varphi_{t}\right\|_{L^{2}\left(\partial E_{\infty}\right)}^{\frac{j}{3}+\frac{n-1}{10}}\left\|\varphi_{t}\right\|_{L^{2}\left(\partial E_{\infty}\right)}^{\frac{3-j}{10}-\frac{n-1}{10}}+C_{F}^{\prime}\left\|\varphi_{t}\right\|_{L^{1}\left(\partial E_{\infty}\right)} \\
& \leq C_{F}^{\prime}\left\|\varphi_{t}\right\|_{H^{3}\left(\frac{3}{3}+\frac{n-1}{10}\right.}^{\frac{3-j}{2}}\left\|\varphi_{t}\right\|_{L^{2}\left(\partial E_{\infty}\right)}^{\frac{3-j}{3}-\frac{n-1}{10}}+C_{F}^{\prime}|\partial F|^{\frac{1}{2}}\left\|\varphi_{t}\right\|_{L^{2}\left(\partial E_{\infty}\right)} \\
& \leq\left(C_{F}^{\prime}\right)^{2}\left\|\psi_{0}\right\|_{H^{3}(\partial F)}^{\frac{1}{2}}\left(e^{-\frac{\sigma_{1}}{2}\left(\frac{3-j}{3}-\frac{n-1}{10}\right) t}+|\partial F|^{\frac{1}{2}} e^{-\frac{\sigma_{1}}{2} t}\right) \\
& \leq\left(1+|\partial F|^{\frac{1}{2}}\right)\left(C_{F}^{\prime}\right)^{2}\left\|\psi_{0}\right\|_{H^{3}(\partial F)}^{\frac{1}{2}} e^{-\frac{\sigma_{1}}{2}\left(\frac{1}{3}-\frac{n-1}{10}\right) t} \\
& \stackrel{(4.5)}{=}\left(1+|\partial F|^{\frac{1}{2}}\right)\left(C_{F}^{\prime}\right)^{2}\left\|\psi_{0}\right\|_{H^{3}(\partial F)}^{\frac{1}{2}} e^{-\sigma_{0} t},
\end{aligned}
$$

which implies that there exists such $C$ as claimed.

Let us finally recall Remark 4.2. In the last step of the previous proof we may replace 5 in the left hand side of (4.11) with any $q \geq 1$ as long as the corresponding $\alpha$ is strictly less than 1 , because $\sigma_{0}=\frac{\eta_{0}}{2}(1-\alpha)$ would then be strictly positive. In the case $n=3$ by replacing 5 with any $1 \leq q<\infty$ we obtain

$$
\alpha=1-\frac{2}{3 q},
$$

so we see that any such $q$ will do. Whereas in the case $n=4$ doing so yields

$$
\alpha=\frac{7}{6}-\frac{1}{q}
$$

and hence $q$ may take any values in the interval $[1,6[$.

\section{APPENDIX}

$C^{1}$ - and $H^{3}$-bounds. In this subsection we prove the estimates (2.10) and (2.11), Lemma 2.8, Lemma 2.9 and Lemma 2.10. We will use the same notation as earlier without any further mention. For sake of simplicity, we use the generic symbol $C$ for a constant which may change from line to line in the estimates but ultimately depends only on $E$.

Let us first fix a smooth set $E$ and let $U_{E}$ be a regular neighborhood of $\partial E$ which we may assume to be of the form $U_{E}=\partial E+B(0, r)$ with some positive $r$. Recall that $\bar{d}_{E}$ and $\pi_{\partial E}$ are $C^{k}$-bounded in $U_{E}$ for every $k \in \mathbb{N}$. For every $\psi \in C^{\infty}(\partial E)$ we set the smooth extension $\psi_{E}=\psi \circ \pi_{\partial E}$. Then $\nabla \psi_{E}=\nabla_{\tau} \psi$ on $\partial E$ and moreover the following decomposition holds on $\partial E$

$$
\mathrm{D}^{2} \psi_{E}=-\nu_{E} \otimes B_{E} \nabla_{\tau} \psi-B_{E} \nabla_{\tau} \psi \otimes \nu_{E}+D_{\tau}^{2} \psi .
$$


For every $\psi \in C^{\infty}(\partial E)$ we set $\Phi_{\psi}: \partial E \rightarrow \mathbb{T}^{n}, \Phi_{\psi}(x)=x+\psi \nu_{E}(x)$, as in Lemma 2.7. We extend $\Phi_{\psi}$ to be the smooth map $U_{E} \rightarrow \mathbb{T}^{n}$ given by $\Phi_{\psi}(x)=x+\psi_{E}(x) \nabla \bar{d}_{E}(x)$. Then

$$
\mathrm{D} \Phi_{\psi}= \begin{cases}I+\psi_{E} \mathrm{D}^{2} \bar{d}_{F}+\nabla \bar{d}_{F} \otimes \nabla \psi_{E} & \text { in } U_{E} \quad \text { and } \\ I+\psi B_{E}+\nu_{E} \otimes \nabla_{\tau} \psi & \text { on } \partial E .\end{cases}
$$

Now $\Phi_{\psi} \rightarrow$ id and $\mathrm{D} \Phi_{\psi} \rightarrow I$ uniformly in $U_{E}$ as $\|\psi\|_{C^{1}(\partial E)} \rightarrow 0$. Thus, by possibly replacing $U_{E}$ with $\partial E+B(0, r / 2), \Phi_{\psi}$ is an orientation preserving diffeomorphism from $U_{E}$ to its image and the set $E_{\psi}$ is well-defined, when $\|\psi\|_{C^{1}(\partial E)}$ is small enough. ${ }^{1}$ The inverse matrix on $\partial E$ is then

$$
\left(\mathrm{D} \Phi_{\psi}\right)^{-1}=\left(I-\nu_{E} \otimes \nabla_{\tau} \psi\right)\left(I+\psi B_{E}\right)^{-1} .
$$

From now on, we assume $\|\psi\|_{C^{1}(\partial E)} \leq \delta$ with $\delta$ small enough so that the previous hold. Further, we use the shorthand notation $A_{\psi}=\left(I+\psi B_{E}\right)^{-1}$ on $\partial E$. Set $u_{\psi}: \Phi_{\psi}\left(U_{E}\right) \rightarrow \mathbb{R}, u_{\psi}=\bar{d}_{E} \circ \Phi_{\psi}^{-1}$. Then $\partial E_{\psi}=u_{\psi}^{-1}(0)$ and $\nu_{E_{\psi}}=\nabla u_{\psi} /\left|\nabla u_{\psi}\right|$ on $\partial E_{\psi}$. Again

$$
\nabla u_{\psi} \circ \Phi_{\psi}=\left(\mathrm{D} \Phi_{\psi}\right)^{-\mathrm{T}} \nu_{E}=\nu_{E}-A_{\psi} \nabla_{\tau} \psi \longrightarrow \nu_{E}
$$

uniformly on $\partial E$ as $\|\psi\|_{C^{1}(\partial E)} \rightarrow 0$. Thus $\nu_{E_{\psi}} \circ \Phi_{\psi}$ also converges uniformly to $\nu_{E}$ as $\psi$ goes to zero in $C^{1}$-sense. The second fundamental form on $\partial E_{\psi}$ can be written, with help of $u_{\psi}$, as

$$
B_{E_{\psi}}=P_{\partial E_{\psi}} \mathrm{D}_{\tau}\left(\frac{\nabla u_{\psi}}{\left|\nabla u_{\psi}\right|}\right)=\frac{1}{\left|\nabla u_{\psi}\right|}\left(I-\nu_{E_{\psi}} \otimes \nu_{E_{\psi}}\right) \mathrm{D}^{2} u_{\psi}\left(I-\nu_{E_{\psi}} \otimes \nu_{E_{\psi}}\right) .
$$

Omitting the details we may further compute that

$$
\mathrm{D}^{2} u_{\psi} \circ \Phi_{\psi}=A_{\psi}\left[B_{E}-\psi\left(\sum_{k=1}^{n}\left\langle\nu_{E}-A_{\psi} \nabla_{\tau} \psi, v_{k}\right\rangle \partial_{v_{k}} \mathrm{D}^{2} \bar{d}_{E}\right)-\mathrm{D}^{2} \psi_{E}\right] A_{\psi}
$$

on $\partial E$, where $v_{1}, \ldots, v_{n}$ is any orthonormal basis of $\mathbb{R}^{n}$. Hence (5.1), (5.2) and (5.3) and the $C^{1}$-bound $\delta$ imply that $\left|\mathrm{D}^{2} u_{\psi} \circ \Phi_{\psi}\right| \leq C\left(1+\left|\mathrm{D}_{\tau}^{2} \psi\right|\right)$ on $\partial E$ with some constant $C$ and so (2.9) holds. Again, by combining the expressions (5.2) and (5.3) we may write on $\partial E$

$$
H_{E_{\psi}} \circ \Phi_{\psi}=\operatorname{tr}\left(Q\left(\cdot, \psi, \nabla_{\tau} \psi\right)\left[B_{E}-\psi\left(\sum_{k=1}^{n}\left\langle\nu_{E}-A_{\psi} \nabla_{\tau} \psi, v_{k}\right\rangle \partial_{v_{k}} \mathrm{D}^{2} \bar{d}_{E}\right)-\mathrm{D}^{2} \psi_{E}\right]\right),
$$

where $Q: \partial E \times[-\delta, \delta] \times[-\delta, \delta]^{n} \rightarrow \mathcal{L}\left(\mathbb{R}^{n} ; \mathbb{R}^{n}\right)$ is a smooth map with $Q(\cdot, 0,0)=P_{\partial E}$. Thus by using Taylor's expansion we may write on $\partial E$

$$
Q\left(\cdot, \psi, \nabla_{\tau} \psi\right)=P_{\partial E}+\psi S\left(\cdot, \psi, \nabla_{\tau} \psi\right)+\left[\left\langle\nabla_{\tau} \psi, r_{i j}\left(\cdot, \psi, \nabla_{\tau} \psi\right)\right\rangle\right]_{i j}
$$

with some smooth $S$ and $r_{i j}$. Thus by substituting (5.5) and (5.1) to (5.4) we obtain the expression (2.8) after regrouping the terms and Lemma 2.8 is clear.

Suppose that $h \in L^{p}\left(\partial E_{\psi}\right)$ with $1 \leq p<\infty$ and $\varphi \in C^{\infty}\left(\partial E_{\psi}\right)$. By using the change of variable formula we have

$$
\begin{aligned}
\int_{\partial E_{\psi}}|h|^{p} \mathrm{~d} \mathcal{H}^{n-1} & =\int_{E}\left|h \circ \Phi_{\psi}\right|^{p} J_{\tau} \Phi_{\psi} \mathrm{d} \mathcal{H}^{n-1} \text { and } \\
\int_{\partial E_{\psi}}\left|\nabla_{\tau} \varphi\right|^{p} \mathrm{~d} \mathcal{H}^{n-1} & =\int_{\partial E_{\psi}}\left|P_{\partial E_{\psi}} \nabla\left(\left(\varphi \circ \Phi_{\psi}\right)_{E} \circ \Phi_{\psi}^{-1}\right)\right|^{p} \mathrm{~d} \mathcal{H}^{n-1} \\
& =\int_{\partial E_{\psi}}\left|P_{\partial E_{\psi}}\left(\mathrm{D} \Phi_{\psi}^{-1}\right)^{\mathrm{T}} \nabla\left(\varphi \circ \Phi_{\psi}\right)_{E} \circ \Phi_{\psi}^{-1}\right|^{p} \mathrm{~d} \mathcal{H}^{n-1} \\
& =\int_{\partial E}\left|\left(I-\nu_{E_{\psi}} \circ \Phi_{\psi} \otimes \nu_{E_{\psi}} \circ \Phi_{\psi}\right)\left(\mathrm{D} \Phi_{\psi}\right)^{-\mathrm{T}} \nabla_{\tau}\left(\varphi \circ \Phi_{\psi}\right)\right|^{p} J_{\tau} \Phi_{\psi} \mathrm{d} \mathcal{H}^{n-1} .
\end{aligned}
$$

Since now $\left(I-\nu_{E_{\psi}} \circ \Phi_{\psi} \otimes \nu_{E_{\psi}} \circ \Phi_{\psi}\right)\left(\mathrm{D} \Phi_{\psi}\right)^{-\mathrm{T}} \rightarrow P_{\partial E}$ and $J_{\tau} \Phi_{\psi} \rightarrow 1$ uniformly on $\partial E$ as $\|\psi\|_{C^{1}(\partial E)}$ tends to zero and $P_{\partial E} \nabla_{\tau}\left(\varphi \circ \Phi_{\psi}\right)=\nabla_{\tau}\left(\varphi \circ \Phi_{\psi}\right)$, then by decreasing $\delta$, if necessary, we find a uniform constant $C$ such that

$$
\begin{gathered}
C^{-1}\left\|h \circ \Phi_{\psi}\right\|_{L^{p}(\partial E)}^{p} \leq\|h\|_{L^{p}\left(\partial E_{\psi}\right)}^{p} \leq C\left\|h \circ \Phi_{\psi}\right\|_{L^{p}(\partial E)}^{p} \quad \text { and } \\
C^{-1-p}\left\|\nabla_{\tau}\left(\varphi \circ \Phi_{\psi}\right)\right\|_{L^{p}(\partial E)}^{p} \leq\left\|\nabla_{\tau} \varphi\right\|_{L^{p}\left(\partial E_{\psi}\right)}^{p} \leq C^{1+p}\left\|\nabla_{\tau}\left(\varphi \circ \Phi_{\psi}\right)\right\|_{L^{p}(\partial E)}^{p},
\end{gathered}
$$

whenever $\|\psi\|_{C^{1}(\partial E)} \leq \delta$. This establishes (2.10) and (2.11). Again, Lemma 2.9 is a direct consequence of (2.10), (2.11) and Remark 2.3.

\footnotetext{
${ }^{1}$ This implies the first part of Lemma 2.7 for smooth functions (the other cases are similar to check). The details of second part are left to the reader.
} 
Since $\left|\partial E_{\psi}\right|$ is now bounded, it is enough to prove Lemma 2.10 for $p=6$. To this end, choose an arbitrary $\varphi \in C^{\infty}\left(\partial E_{\psi}\right)$ and define a smooth extension $\varphi_{E_{\psi}}=\varphi \circ \pi_{\partial E_{\psi}}$ to some regular neighborhood of $\partial E_{\psi}$ as before. A straightforward but a rather long computation yields

$\mathrm{D}_{\tau}^{2}\left(\varphi \circ \Phi_{\psi}\right)=P_{\partial E}\left(\mathrm{D}^{2} \varphi_{E_{\psi}} \circ \Phi_{\psi}\right) \mathrm{D} \Phi_{\psi} P_{\partial E}+\left\langle\nu_{E}, \nabla_{\tau} \varphi \circ \Phi_{\psi}\right\rangle\left(\mathrm{D}_{\tau}^{2} \psi-B_{E}\right)$

$$
\begin{aligned}
& +\psi \sum_{i=1}^{n} P_{\partial E} e_{i} \otimes P_{\partial E}\left(\left(\partial_{i} \mathrm{D} \bar{d}_{E}\right)\left(\nabla_{\tau} \varphi \circ \Phi_{\psi}\right)+\left(\mathrm{D} \Phi_{\psi}\right)^{\mathrm{T}} \mathrm{D}^{2} \varphi_{E_{\psi}} \circ \Phi_{\psi} \partial_{i} \nabla \bar{d}_{E}\right) \\
& +B_{E}\left(\nabla_{\tau} \varphi \circ \Phi_{\psi}\right) \otimes \nabla_{\tau} \psi+\nabla_{\tau} \psi \otimes B_{E}\left(\nabla_{\tau} \varphi \circ \Phi_{\psi}\right)+\nabla_{\tau} \psi \otimes P_{\partial E}\left(\mathrm{D} \Phi_{\psi}\right)^{\mathrm{T}}\left(\mathrm{D}^{2} \varphi_{E_{\psi}} \circ \Phi_{\psi}\right) \nu_{E} .
\end{aligned}
$$

Hence with help of the previous identity

$$
\begin{aligned}
\left|\mathrm{D}_{\tau}^{2}\left(\varphi \circ \Phi_{\psi}\right)\right| & \leq C\left|\mathrm{D}^{2} \varphi_{E_{\psi}} \circ \Phi_{\psi}\right|+\left(C+\left|\mathrm{D}_{\tau}^{2} \psi\right|\right)\left|\nabla_{\tau} \varphi \circ \Phi_{\psi}\right| \\
& \stackrel{(5.1)}{\leq} C\left|\mathrm{D}_{\tau}^{2} \varphi \circ \Phi_{\psi}\right|+C\left(1+\left|B_{E_{\psi}} \circ \Phi_{\psi}\right|+\left|\mathrm{D}_{\tau}^{2} \psi\right|\right)\left|\nabla_{\tau} \varphi \circ \Phi_{\psi}\right| \\
& \stackrel{(2.9)}{\leq} C\left|\mathrm{D}_{\tau}^{2} \varphi \circ \Phi_{\psi}\right|+C\left(1+\left|\mathrm{D}_{\tau}^{2} \psi\right|\right)\left|\nabla_{\tau} \varphi \circ \Phi_{\psi}\right| .
\end{aligned}
$$

Again, Lemma 2.2 is satisfied for $\varphi$ with $p=6, r=q=2, j=1, m=2$ and $n=3$, 4, where $\alpha_{6}=\frac{5}{6}$ for $n=3$ and $\alpha_{6}=1$ for $n=4$. Then

$$
\begin{aligned}
& \left\|\nabla_{\tau} \varphi\right\|_{L^{6}\left(\partial E_{\psi}\right)} \stackrel{(2.11)}{\leq} C\left\|\nabla_{\tau}\left(\varphi \circ \Phi_{\psi}\right)\right\|_{L^{6}(\partial E)} \\
& \stackrel{\text { Lemma }}{\leq} 2.2 C\left\|\mathrm{D}_{\tau}^{2}\left(\varphi \circ \Phi_{\psi}\right)\right\|_{L^{2}(\partial E)}^{\alpha_{6}}\left\|\varphi \circ \Phi_{\psi}\right\|_{L^{2}(\partial E)}^{1-\alpha_{6}} \\
& \leq C\left\|\mathrm{D}_{\tau}^{2}\left(\varphi \circ \Phi_{\psi}\right)\right\|_{L^{2}(\partial E)}+C\left\|\varphi \circ \Phi_{\psi}\right\|_{L^{2}(\partial E)} \\
& \stackrel{(5.6)}{\leq} C\left\|\mathrm{D}_{\tau}^{2} \varphi \circ \Phi_{\psi}\right\|_{L^{2}(\partial E)}+C\left\|\nabla_{\tau} \varphi \circ \Phi_{\psi}\right\|_{L^{2}(\partial E)}+C\left\|\varphi \circ \Phi_{\psi}\right\|_{L^{2}(\partial E)} \\
& +C\left(\int_{\partial E}\left|D_{\tau}^{2} \psi\right|^{2}\left|\nabla_{\tau} \varphi \circ \Phi_{\psi}\right|^{2} \mathrm{~d} \mathcal{H}^{n-1}\right)^{\frac{1}{2}} \\
& (2.10)+(2.11) \\
& \leq C \mathrm{D}_{\tau}^{2} \varphi\left\|_{L^{2}\left(\partial E_{\psi}\right)}+C\right\| \varphi \|_{H^{1}\left(\partial E_{\psi}\right)} \\
& +C\left(\int_{\partial E}\left|D_{\tau}^{2} \psi\right|^{2}\left|\nabla_{\tau} \varphi \circ \Phi_{\psi}\right|^{2} \mathrm{~d} \mathcal{H}^{n-1}\right)^{\frac{1}{2}} \\
& \leq C\left\|\mathrm{D}_{\tau}^{2} \varphi\right\|_{L^{2}\left(\partial E_{\psi}\right)}+C\|\varphi\|_{H^{1}\left(\partial E_{\psi}\right)}+C\left\|D_{\tau}^{2} \psi\right\|_{L^{3}(\partial E)}\left\|\nabla_{\tau} \varphi \circ \Phi_{\psi}\right\|_{L^{6}(\partial E)} \\
& \stackrel{(2.10)}{\leq} C\left\|\mathrm{D}_{\tau}^{2} \varphi\right\|_{L^{2}\left(\partial E_{\psi}\right)}+C\|\varphi\|_{H^{1}\left(\partial E_{\psi}\right)}+C\left\|D_{\tau}^{2} \psi\right\|_{L^{3}(\partial E)}\left\|\nabla_{\tau} \varphi\right\|_{L^{6}\left(\partial E_{\psi}\right)} \\
& \text { (2.5) } \\
& \stackrel{(2.5)}{\leq} C\left\|\Delta_{\tau} \varphi\right\|_{L^{2}\left(\partial E_{\psi}\right)}+C\|\varphi\|_{H^{1}\left(\partial E_{\psi}\right)}+C\left\|D_{\tau}^{2} \psi\right\|_{L^{3}(\partial E)}\left\|\nabla_{\tau} \varphi\right\|_{L^{6}\left(\partial E_{\psi}\right)} \\
& +C\left(\int_{\partial E_{\psi}}\left|B_{E_{\psi}}\right|^{2}\left|\nabla_{\tau} \varphi\right|^{2} \mathrm{~d} \mathcal{H}^{n-1}\right)^{\frac{1}{2}} \\
& \stackrel{(2.10)}{\leq} C\left\|\Delta_{\tau} \varphi\right\|_{L^{2}\left(\partial E_{\psi}\right)}+C\|\varphi\|_{H^{1}\left(\partial E_{\psi}\right)}+C\left\|D_{\tau}^{2} \psi\right\|_{L^{3}(\partial E)}\left\|\nabla_{\tau} \varphi\right\|_{L^{6}\left(\partial E_{\psi}\right)} \\
& +C\left(\int_{\partial E}\left|B_{E_{\psi}} \circ \Phi_{\psi}\right|^{2}\left|\nabla_{\tau} \varphi \circ \Phi_{\psi}\right|^{2} \mathrm{~d} \mathcal{H}^{n-1}\right)^{\frac{1}{2}} \\
& \stackrel{(2.9)}{\leq} C\left\|\Delta_{\tau} \varphi\right\|_{L^{2}\left(\partial E_{\psi}\right)}+C\|\varphi\|_{H^{1}\left(\partial E_{\psi}\right)}+C\left\|D_{\tau}^{2} \psi\right\|_{L^{3}(\partial E)}\left\|\nabla_{\tau} \varphi\right\|_{L^{6}\left(\partial E_{\psi}\right)} \\
& +C\left(\int_{\partial E}\left|\mathrm{D}_{\tau}^{2} \psi\right|^{2}\left|\nabla_{\tau} \varphi \circ \Phi_{\psi}\right|^{2} \mathrm{~d} \mathcal{H}^{n-1}\right)^{\frac{1}{2}}+C\left\|\nabla_{\tau} \varphi \circ \Phi_{\psi}\right\|_{L^{2}(\partial E)} \\
& \leq C\left\|\Delta_{\tau} \varphi\right\|_{L^{2}\left(\partial E_{\psi}\right)}+C\|\varphi\|_{H^{1}\left(\partial E_{\psi}\right)}+C\left\|D_{\tau}^{2} \psi\right\|_{L^{3}(\partial E)}\left\|\nabla_{\tau} \varphi\right\|_{L^{6}\left(\partial E_{\psi}\right)} \\
& +C\left\|D_{\tau}^{2} \psi\right\|_{L^{3}(\partial E)}\left\|\nabla_{\tau} \varphi \circ \Phi_{\psi}\right\|_{L^{6}(\partial E)}+C\left\|\nabla_{\tau} \varphi \circ \Phi_{\psi}\right\|_{L^{2}(\partial E)} \\
& \stackrel{(2.11)}{\leq} C\left\|\Delta_{\tau} \varphi\right\|_{L^{2}\left(\partial E_{\psi}\right)}+C\|\varphi\|_{H^{1}\left(\partial E_{\psi}\right)}+C\left\|D_{\tau}^{2} \psi\right\|_{L^{3}(\partial E)}\left\|\nabla_{\tau} \varphi\right\|_{L^{6}\left(\partial E_{\psi}\right)} \text {. }
\end{aligned}
$$

Again, by Remark $2.3\|\psi\|_{C^{1}\left(\partial E_{\psi}\right)},\left\|\mathrm{D}_{\tau}^{2} \psi\right\|_{L^{3}(\partial E)} \leq C\|\psi\|_{H^{3}\left(\partial E_{\psi}\right)}$, when $n$ is 3 or 4 , so the previous estimate implies Lemma 2.10 in the case $p=6$. 
Time derivatives. In this subsection we derive the formulas (3.25) and (3.26) of Lemma 3.4. In particular, (3.25) is probably well-known, but for sake of completeness we compute it too.

It follows from the semi-group property that we need to check (3.25) and (3.26) at the time $t=0$. At first we list some facts. For that let $\left(E_{t}\right)_{t \in[0, T[}$ be any smooth flow in $\mathbb{T}^{n}$ with a corresponding smoothly parametrized family of diffeomorphism $\left(\Phi_{t}\right)_{t \in[0, T[\text {. Set the initial velocity vector field }}$

$$
X_{0}=\left.\partial_{t} \Phi_{t}\right|_{t=0}
$$

Then $V_{0}=\left\langle X_{0}, \nu_{0}\right\rangle$ and the following hold on the initial boundary $\partial E_{0}$.

$$
\begin{aligned}
\left.\frac{\partial}{\partial t} \nu_{t} \circ \Phi_{t}\right|_{t=0} & =-\left(\mathrm{D}_{\tau} X_{0}\right)^{\mathrm{T}} \nu_{0} \\
\left.\frac{\partial}{\partial t} J_{\tau} \Phi_{t}\right|_{t=0} & =\operatorname{div}_{\tau} X_{0} \quad \text { and } \\
\left.\frac{\partial}{\partial t} H_{t} \circ \Phi_{t}\right|_{t=0} & =-\operatorname{div}_{\tau}\left(\left(\mathrm{D}_{\tau} X_{0}\right)^{\mathrm{T}} \nu_{0}\right)-\operatorname{tr}\left(B_{0} \mathrm{D}_{\tau} X_{0}\right) .
\end{aligned}
$$

For instance, (5.7) is directly computed in [8]. There are also an open neighborhood $U$ of $\bigcup_{t \in[0, T[} \partial E_{t} \times\{t\}$ and a smooth map $H: U \rightarrow \mathbb{T}^{n}$ such that $H(\cdot, t)=H_{t}$ on $\partial E_{t}$ for every $t \in[0, T]$. Again, we recall that every smooth flow admits a normal parametrization, which follows essentially from $[3$, Theorem 8$]$, so we may assume $\left(\Phi_{t}\right)_{t \in[0, T[}$ to be such a parametrization. That is,

$$
\left.\partial_{s} \Phi_{t+s}\right|_{s=0}=\left(V_{t} \circ \Phi_{t}\right)\left(\nu_{t} \circ \Phi_{t}\right)
$$

on $\partial E_{0}$ for every $t \in\left[0, T\left[\right.\right.$, in particular $X_{0}=V_{0} \nu_{0}$ on $\partial E_{0}$. Suppose from now on that $\left(E_{t}\right)_{t}$ is a volume preserving mean curvature flow, so we may write $X_{0}=\left(\bar{H}_{0}-H_{0}\right) \nu_{0}$ and

$$
\mathrm{D}_{\tau} X_{0}=\left(\bar{H}_{0}-H_{0}\right) B_{0}-\nu_{0} \otimes \nabla_{\tau} H_{0}
$$

on $\partial E_{0}$. Hence $(5.7),(5.8)$ and $(5.9)$ can be rewritten as

$$
\begin{aligned}
\left.\frac{\partial}{\partial t} \nu_{t} \circ \Phi_{t}\right|_{t=0} & =\nabla_{\tau} H_{0}, \\
\left.\frac{\partial}{\partial t} J_{\tau} \Phi_{t}\right|_{t=0} & =\left(\bar{H}_{0}-H_{0}\right) H_{0} \text { and } \\
\left.\frac{\partial}{\partial t} H_{t} \circ \Phi_{t}\right|_{t=0} & =\Delta_{\tau} H_{0}-\left(\bar{H}_{0}-H_{0}\right)\left|B_{0}\right|^{2} .
\end{aligned}
$$

The identity (5.13) can be also obtained in a more elegant way using the results from [8]. By using the change of variables formula and integration by parts we compute first

$$
\begin{aligned}
& \left.\frac{\mathrm{d}}{\mathrm{d} t}\left\|\bar{H}_{t}-H_{t}\right\|_{L^{2}\left(\partial E_{t}\right)}^{2}\right|_{t=0}=\left.\frac{\mathrm{d}}{\mathrm{d} t} \int_{\partial E_{0}}\left(\bar{H}_{t} \circ \Phi_{t}-H_{t} \circ \Phi_{t}\right)^{2} J_{\tau} \Phi_{t} \mathrm{~d} \mathcal{H}^{n-1}\right|_{t=0} \\
& =\int_{\partial E_{0}} 2\left(\bar{H}_{0}-H_{0}\right)\left(\left.\frac{\partial}{\partial t} \bar{H}_{t} \circ \Phi_{t}\right|_{t=0}-\left.\frac{\partial}{\partial t} H_{t} \circ \Phi_{t}\right|_{t=0}\right) \mathrm{d} \mathcal{H}^{n-1} \\
& +\left.\int_{\partial E_{0}}\left(\bar{H}_{0}-H_{0}\right)^{2} \frac{\partial}{\partial t} J_{\tau} \Phi_{t}\right|_{t=0} \mathrm{~d} \mathcal{H}^{n-1} \\
& =-\left.2 \int_{\partial E_{0}} \frac{\partial}{\partial t} H_{t} \circ \Phi_{t}\right|_{t=0}\left(\bar{H}_{0}-H_{0}\right) \mathrm{d} \mathcal{H}^{n-1} \\
& +\left.\int_{\partial E_{0}}\left(\bar{H}_{0}-H_{0}\right)^{2} \frac{\partial}{\partial t} J_{\tau} \Phi_{t}\right|_{t=0} \mathrm{~d} \mathcal{H}^{n-1}
\end{aligned}
$$

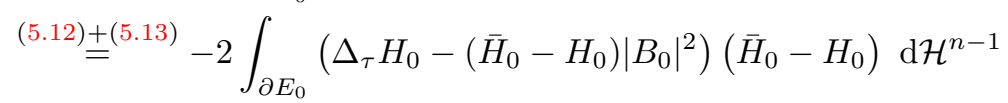

$$
\begin{aligned}
& +\int_{\partial E_{0}} H_{0}\left(\bar{H}_{0}-H_{0}\right)^{3} \mathrm{~d} \mathcal{H}^{n-1} \\
& =-2 \int_{\partial E_{0}}\left|\nabla_{\tau} H_{0}\right|^{2}-\left|B_{0}\right|^{2}\left(\bar{H}_{0}-H_{0}\right)^{2} \mathrm{~d} \mathcal{H}^{n-1} \\
& +\int_{\partial E_{0}} H_{0}\left(\bar{H}_{0}-H_{0}\right)^{3} \mathrm{~d} \mathcal{H}^{n-1} \\
& =-2 \partial^{2} P\left(\partial E_{0}\right)\left[\bar{H}_{0}-H_{0}\right]+\int_{\partial E_{0}} H_{0}\left(\bar{H}_{0}-H_{0}\right)^{3} \mathrm{~d} \mathcal{H}^{n-1} .
\end{aligned}
$$


To compute (3.26) at $t=0$, we evaluate the term $\left.\frac{\partial}{\partial t}\left(\nabla_{\tau} H_{t} \circ \Phi_{t}\right)\right|_{t=0}$ on $\partial E_{0}$. We use the notation $\nabla$ for the spatial gradient. Now

$$
\begin{aligned}
\left.\frac{\partial}{\partial t}\left(\nabla_{\tau} H_{t} \circ \Phi_{t}\right)\right|_{t=0} & =\left.\frac{\partial}{\partial t}\left(I-\nu_{t} \circ \Phi_{t} \otimes \nu_{t} \circ \Phi_{t}\right) \nabla H(\cdot, t) \circ \Phi_{t}\right|_{t=0} \\
& =-\left(\left.\frac{\partial}{\partial t} \nu_{t} \circ \Phi_{t}\right|_{t=0} \otimes \nu_{0}+\left.\nu_{0} \otimes \frac{\partial}{\partial t} \nu_{t} \circ \Phi_{t}\right|_{t=0}\right) \nabla H(\cdot, 0) \\
& +\left.\left(I-\nu_{0} \otimes \nu_{0}\right) \frac{\partial}{\partial t} \nabla H(\cdot, t) \circ \Phi_{t}\right|_{t=0} \\
& \stackrel{(5.11)}{=}-\left\langle\nu_{0}, \nabla H(\cdot, 0)\right\rangle \nabla_{\tau} H_{0}-\left|\nabla_{\tau} H_{0}\right|^{2} \nu_{0} \\
& +\left.\left(I-\nu_{0} \otimes \nu_{0}\right) \frac{\partial}{\partial t} \nabla H(\cdot, t) \circ \Phi_{t}\right|_{t=0}
\end{aligned}
$$

and

$$
\begin{aligned}
\left.\frac{\partial}{\partial t} \nabla H(\cdot, t) \circ \Phi_{t}\right|_{t=0} & =\left.\frac{\partial}{\partial t}\left(\mathrm{D} \Phi_{t}\right)^{-\mathrm{T}} \nabla\left(H(\cdot, t) \circ \Phi_{t}\right)\right|_{t=0} \\
& =\left.\frac{\partial}{\partial t}\left(\mathrm{D} \Phi_{t}\right)^{-\mathrm{T}}\right|_{t=0} \nabla H(\cdot, 0)+\left.\frac{\partial}{\partial t} \nabla\left(H(\cdot, t) \circ \Phi_{t}\right)\right|_{t=0} \\
& =-\left(\left.\mathrm{D} \frac{\partial}{\partial t} \Phi_{t}\right|_{t=0}\right)^{\mathrm{T}} \nabla H(\cdot, 0)+\left.\nabla \frac{\partial}{\partial t}\left(H(\cdot, t) \circ \Phi_{t}\right)\right|_{t=0} \\
& =-\left(\mathrm{D} X_{0}\right)^{\mathrm{T}} \nabla H(\cdot, 0)+\left.\nabla \frac{\partial}{\partial t}\left(H(\cdot, t) \circ \Phi_{t}\right)\right|_{t=0} .
\end{aligned}
$$

By combining the two previous expressions

$$
\begin{aligned}
\left.\frac{\partial}{\partial t}\left(\nabla_{\tau} H_{t} \circ \Phi_{t}\right)\right|_{t=0} & =-\left\langle\nu_{0}, \nabla H(\cdot, 0)\right\rangle \nabla_{\tau} H_{0}-\left|\nabla_{\tau} H_{0}\right|^{2} \nu_{0} \\
& -\left(\mathrm{D}_{\tau} X_{0}\right)^{\mathrm{T}} \nabla H(\cdot, 0)+\left.\nabla_{\tau} \frac{\partial}{\partial t}\left(H(\cdot, t) \circ \Phi_{t}\right)\right|_{t=0} \\
& \stackrel{(5.10)}{=}-\left\langle\nu_{0}, \nabla H(\cdot, 0)\right\rangle \nabla_{\tau} H_{0}-\left|\nabla_{\tau} H_{0}\right|^{2} \nu_{0} \\
& -\left(\left(\bar{H}_{0}-H_{0}\right) B_{0}-\nu_{0} \otimes \nabla_{\tau} H_{0}\right)^{\mathrm{T}} \nabla H(\cdot, 0)+\left.\nabla_{\tau} \frac{\partial}{\partial t}\left(H(\cdot, t) \circ \Phi_{t}\right)\right|_{t=0} \\
& =-\left|\nabla_{\tau} H_{0}\right|^{2} \nu_{0}-\left(\bar{H}_{0}-H_{0}\right) B_{0} \nabla_{\tau} H_{0}+\left.\nabla_{\tau} \frac{\partial}{\partial t}\left(H(\cdot, t) \circ \Phi_{t}\right)\right|_{t=0} \cdot
\end{aligned}
$$

Thus by using the change of variables formula and integrating by parts we finally compute

$$
\begin{aligned}
\left.\frac{\mathrm{d}}{\mathrm{d} t}\left\|\nabla_{\tau} H_{t}\right\|_{L^{2}\left(\partial E_{t}\right)}^{2}\right|_{t=0}= & \left.\frac{\mathrm{d}}{\mathrm{d} t} \int_{\partial E_{0}}\left\langle\nabla_{\tau} H_{t} \circ \Phi_{t}, \nabla_{\tau} H_{t} \circ \Phi_{t}\right\rangle J_{\tau} \Phi_{t} \mathrm{~d} \mathcal{H}^{n-1}\right|_{t=0} \\
= & 2 \int_{\partial E_{0}}\left\langle\left.\frac{\partial}{\partial t} \nabla_{\tau} H_{t} \circ \Phi_{t}\right|_{t=0}, \nabla_{\tau} H_{0}\right\rangle \mathrm{d} \mathcal{H}^{n-1} \\
& +\left.\int_{\partial E_{0}}\left|\nabla_{\tau} H_{t}\right|^{2} \frac{\partial}{\partial t} J_{\tau} \Phi_{t}\right|_{t=0} \mathrm{~d} \mathcal{H}^{n-1} \\
\stackrel{(5.12)}{=} & 2 \int_{\partial E_{0}}\left\langle\left.\frac{\partial}{\partial t} \nabla_{\tau} H_{t} \circ \Phi_{t}\right|_{t=0}, \nabla_{\tau} H_{0}\right\rangle \mathrm{d} \mathcal{H}^{n-1} \\
& +\int_{\partial E_{0}}\left|\nabla_{\tau} H_{t}\right|^{2}\left(\bar{H}_{0}-H_{0}\right) H_{0} \mathrm{~d} \mathcal{H}^{n-1}
\end{aligned}
$$




$$
\begin{aligned}
\stackrel{(5.14)}{=} & 2 \int_{\partial E_{0}}\left\langle-\left(\bar{H}_{0}-H_{0}\right) B_{0} \nabla_{\tau} H_{0}+\left.\nabla_{\tau} \frac{\partial}{\partial t}\left(H(\cdot, t) \circ \Phi_{t}\right)\right|_{t=0}, \nabla_{\tau} H_{0}\right\rangle \mathrm{d} \mathcal{H}^{n-1} \\
& +\int_{\partial E_{0}}\left|\nabla_{\tau} H_{t}\right|^{2}\left(\bar{H}_{0}-H_{0}\right) H_{0} \mathrm{~d} \mathcal{H}^{n-1} \\
= & -\left.2 \int_{\partial E_{0}} \Delta_{\tau} H_{0} \frac{\partial}{\partial t} H_{t} \circ \Phi_{t}\right|_{t=0} \mathrm{~d} \mathcal{H}^{n-1} \\
& -2 \int_{\partial E_{0}}\left(\bar{H}_{0}-H_{0}\right)\left\langle\nabla_{\tau} H_{0}, B_{0} \nabla_{\tau} H_{0}\right\rangle \mathrm{d} \mathcal{H}^{n-1} \\
& +\int_{\partial E_{0}}\left|\nabla_{\tau} H_{t}\right|^{2}\left(\bar{H}_{0}-H_{0}\right) H_{0} \mathrm{~d} \mathcal{H}^{n-1} \\
\stackrel{(5.13)=}{=}- & \int_{\partial E_{0}}\left(\Delta_{\tau} H_{0}\right)^{2}-\left(\bar{H}_{0}-H_{0}\right)\left|B_{0}\right|^{2} \Delta_{\tau} H_{0} \mathrm{~d} \mathcal{H}^{n-1} \\
- & 2 \int_{\partial E_{0}}\left(\bar{H}_{0}-H_{0}\right)\left\langle\nabla_{\tau} H_{0}, B_{0} \nabla_{\tau} H_{0}\right\rangle \mathrm{d} \mathcal{H}^{n-1} \\
& +\int_{\partial E_{0}}\left|\nabla_{\tau} H_{t}\right|^{2}\left(\bar{H}_{0}-H_{0}\right) H_{0} \mathrm{~d} \mathcal{H}^{n-1} .
\end{aligned}
$$

\section{ACKNOWLEDGMENTS}

The author is very thankful to Vesa Julin for many helpful discussions and advice. The work was supported by the Academy of Finland grant 314227.

\section{REFERENCES}

[1] Emilio Acerbi, Nicola Fusco, Vesa Julin, and Massimilliano Morini, Nonlinear stability results for the modified Mullins-Sekerka and the surface diffusion flow, J. Differential Geom. 113 (2019), 1-53.

[2] Emilio Acerbi, Nicola Fusco, and Massimilliano Morini, Minimality via second variation for a nonlocal isoperimetric problem, Comm. Math. Phys. 322 (2013), 515-557.

[3] Luigi Ambrosio and Norman Dancer, Calculus of variations and partial differential equations : topics on geometrical evolution problems and degree theory, Springer-Verlag, Berlin, 2000.

[4] Luigi Ambrosio, Nicola Fusco, and Diego Pallara, Functions of bounded variation and free discontinuity problems, Oxford Mathematical Monographs, Oxford University Press, New York, 2000.

[5] Maria Athanassenas, Behaviour of singularities of the rotationally symmetric, volume-preserving mean curvature flow 17 (2003), 1-16.

[6] Thierry Aubin, Some nonlinear problems in riemannian geometry, Springer Monographs in Mathematics, Springer-Verlag, Berlin, 1998.

[7] J. Lucas Barbosa, Manfredo do Carmo, and Jost Eschenburg, Stability of hypersurfaces of constant mean curvature in Riemannian manifolds, Math. Z. 197 (1988), 123-138.

[8] Filippo Cagnetti, Maria G. Mora, and Massimiliano Morini, A second order minimality condition for the Mumford-Shah functional, Calc. Var. Partial Differential Equations 33 (2008), 37-74.

[9] Joachim Escher and Gieri Simonett, The volume preserving mean curvature flow near spheres, Proc. Amer. Math. Soc. 126 (1998), 2789-2796.

[10] Nicola Fusco, Vesa Julin, and Massimiliano Morini, The surface diffusion flow with elasticity in three dimensions, Arch. Ration. Mech. Anal. 237 (2020), 1325 -1382.

[11] Michael Gage, On an area-preserving evolution equation for plane curves, Contemp. Math. 51 (1986), 51-62.

[12] David Gilbarg and Neil S. Trudinger, Elliptic partial differential equations of second order, Reprint of the 1998 Edition, Classics in Mathematics, Springer-Verlag, Berlin, 2001.

[13] Richard S. Hamilton, Three-manifolds with positive Ricci curvature, J. Differential Geom. 17 (1982), 255-306.

[14] Emmanuel Hebey, Nonlinear analysis on manifolds: Sobolev spaces and inequalities, Courant Lecture Notes in Mathematics, American Mathematical Society, Providence, 2000.

[15] Gerhard Huisken, The volume preserving mean curvature flow, J. Reine Angew. Math. 382 (1987), 35-48.

[16] Haozhao Li, The volume-preserving mean curvature flow in Euclidean space, Pacific J. Math. 243 (2009), 331355.

[17] Uwe F. Mayer and Gieri Simonett, Self-intersections for the surface diffusion and the volume-preserving mean curvature flow, Differential Integral Equations 13 (2000), 1189-1199.

[18] Marty Ross, Schwarz' P and D surfaces are stable, Differential Geom. Appl. 2 (1992), 179-195.

[19] Henry C. Wente, A note on the stability theorem of J. L. Barbosa and M. Do Carmo for closed surfaces of constant mean curvature, Pacific J. Math. 147 (1991), 375-379.

University of Jyvaskyla, Department of Mathematics and Statistics P.O.Box (MaD)FI-40014 UniverSITY OF JYVASKYLA, FINLAND.

Email address: joonas.k.niinikoski@jyu.fi 